\title{
Checklist of Algerian fungi - Part 4: Sordariomycetes (Ascomycota)
}

\author{
Amrani $\mathrm{S}^{1 *}$, Abo Nahas $\mathrm{HH}^{2}$, Ata $\mathrm{TA}^{3}$ and Abdel-Azeem $\mathrm{AM}^{3}$ \\ ${ }^{1 *}$ Laboratoire de Biologie des Sols, Faculté des Sciences Biologiques, Université des Sciences et de la Technologie \\ Houari Boumediène, BP 32 El Alia, Alger 16111, Algeria. \\ ${ }^{2}$ Zoology Department, Faculty of Science, University of Suez Canal, Ismailia 41522, Egypt. \\ ${ }^{3}$ Botany Department, Faculty of Science, University of Suez Canal, Ismailia 41522, Egypt.
}

Amrani S, Abo Nahas HH, Atta TA, Abdel-Azeem AM 2019 - Checklist of Algerian fungi Part 4: Sordariomycetes (Ascomycota). Microbial Biosystems 4(3), 14-54.

\begin{abstract}
A checklist is presented of ascomycetes belonging to class Sordariomycetes in Algeria. This group of fungi is represented in the country by at least 189 genera and 470 species. The list is based on bibliographical, thesis and herbaria online databases sources. This checklist is the first comprehensive species list fully dedicated to Algerian Sordariomycetes.
\end{abstract}

Keywords - Mycobiota, Sordariomyces, biodiversity, literature and herbaria catalogs survey, species list.

\section{Introduction}

Sordariomycetes is the second largest class of Ascomycota (Kirk et al. 2008; Hyde et al. 2013) that involves a highly varied range of fungi characterized mainly by perithecial ascomata and inoperculate unitunicate asci, Lumbsch and Huhndorf (2010) included 18 orders, 63 families and 947 genera, while Kirk et al. (2008) included 15 orders, 64 families, 1119 genera and 10,564 species in Sordariomycetes. The Sordariomycetes have a ecumenical allocation and shelters mostly terrestrial taxa, although several can be found in aquatic habitats (Hyde and Wong 2000; Samuels and Blackwell 2001; Cai et al. 2006; Jones et al. 2009, 2015; Pratibha et al. 2014).

Species in this order dwell in different habitats they can be pathogens, saprobes, endophytes or epiphytes (Zhang et al. 2006; Kirk et al. 2008). Some are phytopathogens that cause leaf, stem and root diseases on a vast variety of hosts, while some provoke diseases in arthropods and mammals (Sung et al. 2007; Prados-Rosale et al. 2012; Hyde et al. 2014). Sordariomycetes are usually isolated as endophytes from several plants (Keim et al. 2014) many exist as saprobes involved in nutrient and decomposition cycling (Jaklitsch and Voglmayr 2012) as some taxa are fungicolous (Pei Gui et al. 2000), some species produce a vast range of chemically assorted metabolites highly signigicant in medicinal and other biotechnological industries (Semenova et al. 2012; Debbab et al. 2013; Xu et al. 2014) and other species of Sordariomycetes such as Beauveria bassiana, Trichoderma viride, T. harzianum are economically important biocontrol agents (Wraight et al.1998; Kaewchai et al. 2009). 
For a large country like Algeria no published checklist concerning Sordariomycetes was found up till now. We trust that this checklist will encourage scientists to investigate an important component of Algeria's biodiversity and to make it better known to the world's scientific community.

\section{Material and Methods}

\section{Study area}

Algeria, ca. 2.4 million $\mathrm{km}^{2}$ in area, is the largest country of Africa since the partition of Sudan in 2011. For more details about the study area please check Amrani and Abdel-Azeem (2019).

\section{Data Collection.}

The species listed here were compiled from bibliographical and herbaria online databases sources. A main list of Algerian Laboulbeniales has been developed and the taxa are given in alphabetical sequence of taxa and accepted names are highlighted in bold . The names of authors of fungal taxa are abbreviated according to Kirk and Ansell (1992) and Kirk et al. (2008). Name corrections, authorities reported in this work were checked against the databases of Index fungorum (www.indexfungorum.org) and Mycobank (www.mycobank.org).

\section{Checklist of Algerian Sordariomycetes}

\section{Abbreviations}

GBIF: Global Biodiversity Information Facility (https://www.gbif.org)

CABI: Centre for Agricultural Bioscience International (https://www.cabi.org)

\section{Cited herbaria (put inside square brackets in the text)}

BPI: United States National Fungus Collections, Beltsville, USA - BR: Meise Botanic Garden, Meise, Belgium - BRLU: Herbarium de l'Université Libre de Bruxelles, Belgium - CUP: Cornell University, Plant Pathology Herbarium, New York, USA - FH: Farlow Herbarium, Harvard University, Cambridge, USA - G: Conservatoire et Jardin botaniques de la Ville de Genève, Geneve, Switzerland - H: Finnish Museum of Natural History (University of Helsinki), Helsinki, Finland - ILL: University of Illinois Herbarium, Champaign, USA - MA: Real Jardín Botánico , Madrid, Spain - MIN: University of Minnesota, Bell Museum of Natural History Herbarium Fungal Collection, St Paul, USA - MPU: Université Montpellier, Montpellier, France - NEB: University of Nebraska State Museum, Lincoln, USA - NY: New York Botanical Garden, New York, USA - P: Muséum National d'Histoire Naturelle, Paris, France - PUR: Purdue University, Kriebel Herbarium, Lafayette, USA - S: Swedish Museum of Natural History (Naturhistoriska riksmuseet), Stockholm, Sweden - UPS: The Museum of Evolution Herbarium, Uppsala, Sweden.

\section{Diaporthomycetidae}

1. Amphiporthe leiphaemia (Fr.) Butin (syn. Discula quercina) Bensaci (2017) unpublished (retrieved from NCBI nucleotide).

2. Apiognomonia veneta (Sacc. \& Speg.) Höhn. Kedad and Bouznad (2018). 
3. Brachysporium urophorum (Durieu \& Mont.) Sacc.

Saccardo (1886).

4. Breviappendix rostellata (Fr.) Senan. \& K.D. Hyde (syn. Gnomonia rostellata, Sphaeria rostellata)

Durieu (1846), Kedad and Bouznad (2018), [P].

5. Cryphonectria naterciae Bragança, E. Diogo \& A.J.L. Phillips Smahi et al. (2018).

6. Cryptosporella hypodermia (Fr.) Sacc. (syn. Sphaeria hypodermia, Winterella hypodermia)

Durieu (1846), Kedad and Bouznad (2018), [P].

7. Diaporthe ampelina (Berk. \& M.A. Curtis) R.R. Gomes, Glienke \& Crous Larignon (2016).

8. Diaporthe chamaeropis (Cooke) R.R. Gomes (syn. Phoma chamaeropis, Phomopsis chamaeropis).

[UPS].

9. Diaporthe conjuncta (Nees) Fuckel (syn. Sphaeria conjuncta)

Durieu (1846), Kedad and Bouznad (2018), [P].

10. Diaporthe eumorpha (Durieu \& Mont.) Maire

Maire (1917), Kedad and Bouznad (2018), [BPI].

11. Diaporthe hemicrypta (Durieu \& Mont.) Sacc. (syn. Sphaeria hemicrypta) Durieu (1846), Montagne (1856), Saccardo (1883 \& 1891), Kedad and Bouznad (2018), $[\mathrm{P}]$

12. Diaporthe lirelliformis Pat.

Patouillard (1897a), Saccardo (1899), Pfister (1977), Kedad and Bouznad (2018).

13. Diaporthe neoviticola Udayanga, Crous \& K.D. Hyde (syn. Phomopsis viticola). Berraf and Péros (2005).

14. Diaporthe spiculosa (Westend.) Nitschke (syn. Sphaeria spiculosa)

Durieu (1846), Kedad and Bouznad (2018), [P].

15. Diaporthe syngenesia (Fr.) Fuckel (syn. Sphaeria syngenesia)

Durieu (1846), Kedad and Bouznad (2018), [P].

\section{Hypocreomycetidae}

16. Acanthonitschkea tristis (J. Kickx f.) Nannf. (syn. Coelosphaeria tristis) Saccardo (1882), Kedad and Bouznad (2018).

17. Acremonium masseei (Sacc.) W. Gams $[\mathrm{P}]$

18. Acremonium persicinum (Nicot) W. Gams (syn. Paecilomyces persicinus) Dubost (1966). 


\section{Acremonium potronii Vuill.}

Catanei $(1934,1938)$.

20. Acremonium rutilum W. Gams (syn. A. roseum) Chafai (1996).

21. Acrostalagmus luteoalbus (Link) Zare, W. Gams \& Schroers (syn. A. cinnabarinus, Botrytis lateritia, Sporotrichum luteo).

Durieu (1846), Killian and Fehér (1935), Kedad and Bouznad (2018), [P].

\section{Aecidium alaterni Maire} Maire (1900), Juel (1901), Sydow and Sydow (1915), Kedad and Bouznad (2018), [S].

23. Akanthomyces lecanii (Zimm.) Spatafora, Kepler \& B. Shrestha (syn. Cordyceps confragosa).

Vu et al. (2019).

24. Albifimbria verrucaria (Alb. \& Schwein.) L. Lombard \& Crous (syn. Myrothecium verrucaria).

Nicot (1955), Dubost (1966), Locquin-Linard (1988), Chamekh et al. (2019).

25. Aleurisma lugdunense Vuill. Ainsworth and Austwick (1959).

26. Allantonectria miltina (Durieu \& Mont.) Weese (syn. Nectria miltina, Nectriella miltina, Sphaeria miltina).

Durieu (1846), Montagne (1856), Saccardo (1878, 1883), Kedad and Bouznad (2018), $[\mathrm{P}]$.

27. Beauveria bassiana (Bals.-Criv.) Vuill. (syn. Sporotrichum globuliferum). Trabut (1898), Zamoum et al. (2011), Abdelaziz et al. (2018), Kedad and Bouznad (2018), Chamekh et al. (2019).

28. Beauveria brongniartii (Sacc.) Petch (syn. Botrytis brongniartii)

Saccardo (1892), Maire (1906), Kedad and Bouznad (2018).

29. Beauveria felina (DC.) J.W. Carmich. (syn. Amphichorda felina, Isaria felina) Faurel and Schotter (1964a), [P].

30. Bertia moriformis (Tode) De Not. (syn. Sphaeria moriformis) Durieu (1846), Maire and Werner (1938), Kedad and Bouznad (2018).

31. Bisifusarium dimerum (Penz.) L. Lombard \& Crous (syn. Fusarium dimerum) Dubost (1966).

32. Cephalotrichum purpureofuscum (S. Hughes) S. Hughes (syn. Doratomyces purpureofuscus). Locquin-Linard (1988).

33. Cephalotrichum stemonitis (Pers.) Nees (syn. Periconia stemonitis) Durieu (1846), Kedad and Bouznad (2018).

34. Ceratocystis paradoxa (Dade) C. Moreau Kedad and Bouznad (2018). 
35. Chadefaudia corallinarum (P. Crouan \& H. Crouan) E. Müll. \& Arx (syn. Physalospora corallinarum).

Kohlmeyer (1963).

36. Chadefaudia gymnogongri (Feldmann) Kohlm. (syn. Mycophycophila gymnogongri, Macrophoma gymnogongri)

Feldmann (1940), Kohlmeyer (1963), , CMI (1954).

37. Chadefaudiella quezelii Faurel \& Schotter

CMI (1972).

38. Chadefaudiella thomasii Faurel \& Locq.

Benny and Kinbrough (1980), CMI (1981).

39. Chaetosphaerella fusca (Fuckel) E. Müll. \& C. Booth (syn. Cladotrichum polysporum) Durieu (1846), Kedad and Bouznad (2018), [P].

40. Chaetosphaerella phaeostroma (Durieu \& Mont.) E. Müll. \& C. Booth (syn. Sphaeria phaeostroma)

Durieu (1846), Montagne (1856), Saccardo (1883), Kedad and Bouznad (2018), [P].

41. Chalara microspora (Corda) S. Hughes (syn. Fusidium clandestinum var. microsporum) $[\mathrm{P}]$.

42. Claviceps purpurea (Fr.) Tul. (syn. C. microcephala, Sclerotium clavus, Sphacelia segetum).

Durieu (1846), Saccardo and Berlese (1886), Roumeguère (1887), Maire (1916), Kedad and Bouznad (2018), [BPI], [BR], [NEB], [NY], [PUL].

43. Clonostachys rosea (Link) Schroers, Samuels, Seifert \& W. Gams (syn. Gliocladium roseum).

Maire and Werner (1938), Kahoul et al. (2014), Kedad and Bouznad (2018), Chamekh et al. (2019).

44. Colletotrichum bryoniae (Ferraris) Maire

Maire (1917), Saccardo (1931), Kedad and Bouznad (2018), [MPU].

45. Colletotrichum circinans (Berk.) Voglino

Kedad and Bouznad (2018).

46. Colletotrichum coccodes (Wallr.) S. Hughes (syn. Gloeosporium olivarum) Stevenson (1926).

47. Colletotrichum dematium (Pers.) Grove (syn. Vermicularia dematium) Durieu (1846), Maire and Werner (1938), Kedad and Bouznad (2018), [BR]

48. Colletotrichum gloeosporioides (Penz.) Penz. \& Sacc.

Mahiout et al. (2019).

49. Colletotrichum liliacearum (Westend.) Duke (syn. Vermicularia liliacearum) Maire (1906), Kedad and Bouznad (2018).

50. Colletotrichum lindemuthianum (Sacc. \& Magnus) Briosi \& Cavara Kedad and Bouznad (2018). 


\section{Colletotrichum tetrapanacis Maire}

[MPU].

52. Colletotrichum trichellum (Fr.) Duke (syn. Vermicularia arophila, V. trichella subsp. arophila).

Maire (1906), Kedad and Bouznad (2018).

53. Corallomycetella repens (Berk. \& Broome) Rossman \& Samuels (syn. Nectria mauritiicola).

Belhoucine et al. (2012), Kedad and Bouznad (2018).

54. Cordyceps tuberculata (Lebert) Maire (syn. C. sphingum)

Lagarde (1913-14), Maire (1917), Kedad and Bouznad (2018).

55. Cylindrium aeruginosum (Link) Lindau

Maire (1906), Kedad and Bouznad (2018).

56. Cylindrium elongatum Bonord.

Maire (1906), Kedad and Bouznad (2018).

57. Cylindrium flavovirens (Ditmar) Bonord. (syn. Fusidium flavovirens)

Durieu (1846), Kedad and Bouznad (2018).

58. Dactylonectria macrodidyma (Halleen, Schroers \& Crous) L. Lombard \& Crous Aigoun-Mouhous et al. (2019).

59. Dactylonectria novozelandica (A. Cabral \& Crous) L. Lombard \& Crous Aigoun-Mouhous et al. (2019).

60. Dactylonectria torresensis (A. Cabral, Rego \& Crous) L. Lombard \& Crous Aigoun-Mouhous et al. (2019).

61. Dialonectria episphaeria (Tode) Cooke (syn. Sphaeria episphaeria, S. sanguinea) Durieu (1846), Maire (1906), Kedad and Bouznad (2018), [P].

62. Emericellopsis terricola J.F.H. Beyma

Kahoul et al. (2014), [P].

63. Enterocarpus grenotii Locq.-Lin. Locquin-Linard (1977, 1988), [P].

64. Enterocarpus uniporus Locq.-Lin.

Locquin-Linard $(1977,1988)$.

65. Epichloe typhina (Pers.) Tul. \& C. Tul., Maire (1919), Kedad and Bouznad (2018), [BPI], [MA].

66. Faurelina fimigena Locq.-Lin. Locquin-Linard (1988).

67. Furcasterigmium furcatum (Moreau \& V. Moreau ex W. Gams) Giraldo López \& Crous (syn. Acremonium furcatum).

Bekhouche et al. (1994). 
68. Fusarium acuminatum Ellis \& Everh.

Lazreg et al. (2013a).

69. Fusarium algeriense Laraba \& O'Donnell

Laraba et al. (2018).

70. Fusarium avenaceum (Fr.) Sacc.

Kachour (2004), Kedad and Bouznad (2018).

71. Fusarium brachygibbosum Padwick

Chamekh et al. (2019).

72. Fusarium chlamydosporum Wollenw. \& Reinking Lazreg (2015), Lazreg et al. (2013c, 2014b), [P].

73. Fusarium cicatricum (Berk.) O'Donnell \& Geiser (syn. Nectria desmazieri) Saccardo and Berlese (1886), Kedad and Bouznad (2018).

74. Fusarium commune K. Skovg., O'Donnell \& Nirenberg

Edel-Hermann et al. (2011).

75. Fusarium culmorum (Wm.G. Sm.) Sacc.

Dubost (1966), Bekhouche et al. (1994), Kachour (2004), Laraba et al. (2018), Kedad and Bouznad (2018).

76. Fusarium equiseti (Corda) Sacc. Lazreg (2015), Lazreg et al. (2014a), Chamekh et al. (2019), Yezli et al. (2019).

77. Fusarium fujikuroi Nirenberg (syn. F. moniliforme, F. verticillioides). Boutaiba (1997).

78. Fusarium graminearum Schwabe (syn. Dichomera saubinetii, Gibbera saubinetii, Gibberella saubinetii). Montagne (1856), Saccardo (1883), Kedad and Bouznad (2018), [MPU].

79. Fusarium lolii (Wm.G. Sm.) Sacc. (syn. F. heterosporum). Saccardo and Berlese (1886), Kedad and Bouznad (2018).

80. Fusarium oxysporum f.sp. albedinis W.L. Gordon Kedad and Bouznad (2018).

81. Fusarium oxysporum f.sp. ciceris Matuo \& K. Satô Tlemsani (2010), Kedad and Bouznad (2018).

82. Fusarium oxysporum f.sp. lentis W.L. Gordon Kedad and Bouznad (2018).

83. Fusarium oxysporum f.sp. lycopersici W.C. Snyder \& H.N. Hansen Kedad and Bouznad (2018).

84. Fusarium oxysporum f.sp. melonis W.C. Snyder \& H.N. Hansen Kedad and Bouznad (2018).

85. Fusarium oxysporum f.sp. pisi W.C. Snyder \& H.N. Hansen Kedad and Bouznad (2018). 
86. Fusarium oxysporum f.sp. radicis-lycopersici Jarvis \& Shoemaker Lakhdari (2017).

87. Fusarium oxysporum Schltdl. (syn. F. albedinis, F. aurantiacum, F. lini). Durieu (1846), Maire and Werner (1938), Bekhouche et al. (1994), Chafai (1996), Boutaiba (1997), Kachour (2004), Belyagoubi (2006), Edel-Hermann et al. (2011), Lazreg (2015), Kahoul et al. (2014), Lazreg et al. (2014b), Mohamed-Mahmoud et al. (2016), Benzohra and Megateli (2017), Abdelaziz et al. (2018), Bourzama et al. (2018), Kedad and Bouznad (2018), Chamekh et al. (2019).

\section{Fusarium pallens Nees}

$[\mathrm{P}]$.

89. Fusarium proliferatum (Matsush.) Nirenberg ex Gerlach \& Nirenberg $[\mathrm{P}]$.

90. Fusarium pseudograminearum O'Donnell \& T. Aoki Kedad and Bouznad (2018).

91. Fusarium punctiforme Durieu \& Mont. Durieu (1846), Saccardo (1882, 1886), Kedad and Bouznad (2018), [P].

92. Fusarium redolens Wollenw. Edel-Hermann et al. (2011), Lazreg et al. (2013b, 2014b), Lazreg (2015).

93. Fusarium roseum Link (syn. Gibberella pulicaris, Sphaeria pulicaris). Durieu (1846), Saccardo (1878, 1883), Kedad and Bouznad (2018), [P]

94. Fusarium sporotrichioides Sherb. Kedad and Bouznad (2018).

95. Fusarium stictoides Durieu \& Mont. Durieu (1846), Montagne (1856), Kedad and Bouznad (2018), [P].

96. Fusarium trichothecioides Wollenw. Kahoul et al. (2014).

97. Fusarium tricinctum (Corda) Sacc. Lazreg et al. (2014b), Lazreg (2015).

98. Fusidium griseum Ditmar ex Link Durieu (1846), Kedad and Bouznad (2018).

99. Fusidium verrucosum Durieu \& Mont.

Durieu (1846), Montagne (1856), Saccardo (1886), Kedad and Bouznad (2018), [P].

100. Fusisporium erubescens Durieu \& Mont.

Durieu (1846), Montagne (1856), Saccardo (1886), Kedad and Bouznad (2018), [P].

101. Geosmithia langdonii M. Kolarík, Kubátová \& Pazoutová

Belhoucine et al. (2012), Kedad and Bouznad (2018).

102. Gibberella acervalis (Moug.) Sacc.

Durieu (1846), Kedad and Bouznad (2018), [P]. 
103. Gibellulopsis nigrescens (Pethybr.) Zare, W. Gams \& Summerb.

Chamekh et al. (2019).

104. Gliomastix luzulae (Fuckel) E.W. Mason ex S. Hughes

Locquin-Linard (1988).

105. Graphium berkeleyi Mont.

Montagne (1857).

106. Graphium penicillioides Corda [MPU].

107. Graphium rigidum (Pers.) Sacc. (syn. Stilbum rigidum).

Durieu (1846), Kedad and Bouznad (2018).

108. Hyalopus curtipes (Sacc.) M.A.J. Barbosa (syn. Cephalosporium curtipes). Dubost (1966).

109. Hypomyces aurantius (Pers.) Fuckel (syn. Diplocladium minus, Sphaeria aurantia). Durieu (1846), Maire (1917), Kedad and Bouznad (2018), [MPU], [P].

110. Hypomyces chrysospermus Tul. \& C. Tul. (syn. Sepedonium chrysospermum). Durieu (1846), Kedad and Bouznad (2018).

111. Hypomyces lithuanicus Heinr.-Norm. Rogerson and Samuels (1994).

112. Hypomyces rosellus (Alb. \& Schwein.) Tul. \& C. Tul. (syn. Sphaeria rosella var. haematea).

Durieu (1846), Kedad and Bouznad (2018).

113. Hypomyces torminosus (Mont.) Tul. \& C. Tul. (syn. Nectria torminosa, Sphaeria torminosa).

Durieu (1846), Montagne (1856), Saccardo (1878, 1883), Kedad and Bouznad (2018), $[\mathrm{P}]$.

114. Illosporium maculicola Sacc. [MPU].

115. Illosporium roseum Mart. (syn. Illosporium carneum). Faurel and Schotter (1964a).

116. Kernia nitida (Sacc.) Nieuwl. (syn. Magnusia nitida). Faurel and Schotter (1964b), Locquin-Linard (1988).

117. Kernia setadisperma Locq.-Lin. Locquin-Linard (1988).

118. Lecanicillium lecanii (Zimm.) Zare \& W. Gams (syn. Cephalosporium lecanii). [MPU].

119. Lecanicillium psalliotae (Treschew) Zare \& W. Gams Kedad and Bouznad (2018). 
120. Lophotrichus ampullus R.K. Benj.

Locquin-Linard (1988).

121. Lophotrichus bartlettii (Massee \& E.S. Salmon) Malloch \& Cain (syn. Magnusia bartletti).

Faurel and Schotter (1964b).

122. Lophotrichus macrosporus (Faurel \& Locq.-Lin.) Arx, Figueras \& Guarro (syn. Kernia macrospora).

Locquin-Linard (1988), [P].

123. Melanopsamma glandis (Duby) Sacc. (syn. Sphaeria glandis).

Durieu (1846), Saccardo (1882), Kedad and Bouznad (2018).

124. Melanopsamma pomiformis (Pers.) Sacc.

Saccardo (1882), Kedad and Bouznad (2018).

125. Melanospora camelina Faurel \& Schotter

Faurel and Schotter (1965).

126. Melanospora zobelii (Corda) Fuckel [MPU].

127. Metarhizium anisopliae (Metschn.) Sorokin

Benserradj and Mihoubi (2014).

128. Metarhizium flavoviride W. Gams \& Rozsypal Abdelaziz et al. (2018).

129. Metarhizium marquandii (Massee) Kepler, S.A. Rehner \& Humber (syn. Spicaria violacea).

Dubost (1966).

130. Microascus appendiculatus Woudenb. \& Samson

Woudenberg et al. (2017).

131. Microascus chartarum (G. Sm.) Sandoval-Denis, Gené \& Guarro (syn. Scopulariopsis chartarum).

Bekhouche et al. (1994).

132. Microascus cirrosus Curzi (syn. Microascus cirrhosus).

$[\mathrm{P}]$.

133. Microascus croci (J.F.H. Beyma) Sandoval-Denis, Gené \& Guarro (syn. Scopulariopsis croci).

$[\mathrm{P}]$.

134. Microascus manginii (Loubière) Curzi

Chamekh et al. (2019).

135. Microascus senegalensis Arx Jagielski et al. (2016).

136. Microascus trigonosporus C.W. Emmons \& B.O. Dodge Dubost (1966), Locquin-Linard (1988), Bekhouche et al. (1994), [P]. 
137. Microcera larvarum (Fuckel) Gräfenhan, Seifert \& Schroers (syn. M. parlatoriae, Fusarium larvarum).

Trabut (1907), Bensaci et al. (2015).

138. Monocillium loricatum W. Gams

[P], CMI (1981).

139. Myrothecium inundatum Tode

$[\mathrm{P}]$.

140. Myrothecium pygmaeum Faurel \& Schotter

Faurel and Schotter (1965).

141. Nectria cesatii Mont.

Montagne (1857).

142. Nectria cinnabarina (Tode) Fr. (syn. Sphaeria cinnabarina, S. ochracea fries, Tubercularia vulgaris).

Durieu (1846), Kedad and Bouznad (2018), [P].

143. Nectria peziza (Tode) Fr. (syn. Sphaeria peziza).

Durieu (1846), Kedad and Bouznad (2018), [P].

144. Nectria pyrosphaera Maire

Maire (1917, 1927), Saccardo (1926), Kedad and Bouznad (2018), [MA], [MPU], [NY].

145. Neocosmospora solani (Mart.) L. Lombard \& Crous (syn. Fusarium solani).

Locquin-Linard (1988), Bekhouche et al. (1994), Chafai (1996), Kahoul et al. (2014), Lazreg (2015), Lazreg et al. (2014b), Kedad and Bouznad (2018).

146. Neonectria coccinea (Pers.) Rossman \& Samuels (syn. Nectria coccinea). Saccardo and Berlese (1886), Kedad and Bouznad (2018).

147. Nigrosabulum globosum Malloch \& Cain

Locquin-Linard (1988), [P].

148. Ophiocordyceps gracilis (Grev.) G.H. Sung, J.M. Sung, Hywel-Jones \& Spatafora (syn. Cordyceps gracilis).

Durieu (1846), Montagne (1856), Saccardo (1878, 1883), Kedad and Bouznad (2018), $[\mathrm{P}]$.

149. Ophionectria mellina (Durieu \& Mont.) Sacc. (syn. Nectria mellina, Sphaeria mellina). Durieu (1846), Montagne (1856), Saccardo (1878, 1883), Kedad and Bouznad (2018).

150. Ophiostoma nigrocarpum (R.W. Davidson) de Hoog Kedad and Bouznad (2018).

151. Paramyrothecium roridum (Tode) L. Lombard \& Crous (syn. Myrothecium roridum). Durieu (1846), Bekhouche et al. (1994), Kedad and Bouznad (2018).

152. Parengyodontium album (Limber) C.C. Tsang et al. (syn. Tritirachium album). Dubost (1966). 
153. Pleiocarpon algeriense W. Aigoun-Mouhous, A. Cabral and A. Berraf-Tebbal (syn. Pleiocarpon algeriense).

Aigoun-Mouhous et al. (2019).

154. Pseudallescheria boydii (Shear) McGinnis, A.A. Padhye \& Ajello (syn. Allescheria boydii, Aleurisma apiospermum, Scedosporium apiospermum).

Montpellier and Gouillon (1921), Montpelier and Catanei (1927), Catanei and Legroux (1931), Catanei (1934, 1938).

155. Pseudonectria buxi (DC.) Seifert, Gräfenhan \& Schroers (syn. Volutella buxi). Saccardo and Roumeguère (1881), Kedad and Bouznad (2018).

156. Purpureocillium lilacinum (Thom) Luangsa-ard, Houbraken, Hywel-Jones \& Samson (syn. Paecilomyces lilacinus).

Locquin-Linard (1988), Chamekh et al. (2019).

157. Rectifusarium ventricosum (Appel \& Wollenw.) L. Lombard \& Crous (syn. Fusarium ventricosum).

$[\mathrm{P}]$.

158. Sarocladium strictum (W. Gams) Summerb. (syn. Acremonium strictum, Cephalosporium acremonium).

Killian and Fehér (1935), Locquin-Linard (1988), Bekhouche et al. (1994), Chamekh et al. (2019).

159. Scopinella barbata (Pers.) Lév. (syn. Melanospora barbata). Durieu (1846), Saccardo (1878), Saccardo (1883), Kedad and Bouznad (2018).

160. Scopulariopsis arnoldii (L. Mangin \& Pat.) Vuill. (syn. Monilia arnoldi). Saccardo (1913), Pfister (1977), Kedad and Bouznad (2018).

161. Simplicillium lamellicola (F.E.V. Sm.) Zare \& W. Gams (syn. Verticillium lamellicola).

$[\mathrm{P}]$.

162. Stachybotrys chartarum (Ehrenb.) S. Hughes (syn. Stachybotrys atra, S. lobulata) Maire (1917), Faurel and Schotter (1964b), Lunghini (1978), Locquin-Linard (1988), Bekhouche et al. (1994), Kedad and Bouznad (2018), [P].

163. Stachybotrys echinatus (Rivolta) G. Sm. (syn. Haplographium echinatum, Memnoniella echinata).

$[\mathrm{P}]$.

164. Stilbella fimetaria (Pers.) Lindau (syn. Stilbella erythrocephala).

Locquin-Linard (1988).

165. Syspastospora parasitica (Tul.) P.F. Cannon \& D. Hawksw. (syn. Melanospora parasitica).

Patouillard (1903), Kedad and Bouznad (2018).

166. Tolypocladium ophioglossoides (J.F. Gmel.) C.A. Quandt, Kepler \& Spatafora (syn. Cordyceps ophioglossoides).

[BPI]. 
167. Trichoderma asperellum Samuels, Lieckf. \& Nirenberg Kedad and Bouznad (2018).

168. Trichoderma atroviride P. Karst. Kedad and Bouznad (2018).

169. Trichoderma aureoviride Rifai Benzohra and Megateli (2017).

170. Trichoderma gamsii Samuels \& Druzhin. Chamekh et al. (2019).

171. Trichoderma hamatum (Bonord.) Bainier Chafai (1996).

172. Trichoderma harzianum Rifai Chafai (1996), Kahoul et al. (2014), Benzohra and Megateli (2017), Kedad and Bouznad (2018).

173. Trichoderma hispanicum (Jaklitsch \& Voglmayr) Jaklitsch \& Voglmayr Chamekh et al. (2019).

174. Trichoderma koningii Oudem. Killian and Fehér (1935), Chafai (1996), Kahoul et al. (2014), Chamekh et al. (2019).

175. Trichoderma koningiopsis Samuels, Carm. Suárez \& H.C. Evans Chamekh et al. (2019).

176. Trichoderma lixii (Pat.) P. Chaverri Kedad and Bouznad (2018).

\section{Trichoderma longibrachiatum Rifai} Benzohra and Megateli (2017), Kedad and Bouznad (2018).

178. Trichoderma orientale (Samuels \& Petrini) Jaklitsch \& Samuels Bekkar (2017) Unpublished, Lehad A. (2019) Unpublished (retrieved from NCBI nucleotide).

179. Trichoderma patella (Cooke \& Peck) Jaklitsch \& Voglmayr (syn. Hypocrea patella). Maire (1917), Kedad and Bouznad (2018), [MPU].

180. Trichoderma polysporum (Link) Rifai (syn. Sporotrichum polysporum). Killian and Fehér (1935)

181. Trichoderma saturnisporopsis Samuels \& Jaklitsch Kahoul et al. (2014).

182. Trichoderma viride Pers. (syn. Hypocrea rufa) Durieu (1846), Maire (1906), Killian and Fehér (1935), Nicot (1955), Dubost (1966), Locquin-Linard (1988), Chafai (1996), Abdelaziz et al. (2018), Bourzama et al. (2018), Kedad and Bouznad (2018), [P].

183. Tubercularia leucoloma Durieu \& Mont. Durieu (1846), Kedad and Bouznad (2018). 
184. Tubercularia phacidioides Durieu \& Mont.

Durieu (1846), Kedad and Bouznad (2018).

185. Tuberculina phacidioides (Durieu \& Mont.) Sacc.

Saccardo (1886).

186. Vermicularia culmigena Desm.

Saccardo and Berlese (1886), Kedad and Bouznad (2018).

187. Vermicularia duriaei (Mont.) Mont.

Durieu (1846), Kedad and Bouznad (2018).

188. Vermicularia ephedrae Durieu \& Mont.

Durieu (1846), Saccardo and Roumeguère (1881), Saccardo (1884), Patouillard (1903),

Kedad and Bouznad (2018).

189. Vermicularia macrochaeta Desm.

Durieu (1846), Kedad and Bouznad (2018).

190. Verticillium africanum (Durieu \& Mont.) Sacc. (syn. Botrytis africana).

Saccardo (1886), Kedad and Bouznad (2018).

191. Verticillium alfalfae Inderb., H.W. Platt, R.M. Bostock, R.M. Davis \& Subbarao (syn. Verticillium alfalfae).

Abdelaziz et al. (2018).

192. Verticillium dahliae Kleb.

Chliyeh et al. (2014), Kedad and Bouznad (2018).

193. Volutella ciliata (Alb. \& Schwein.) Fr. (syn. Chaetostroma stipitatum).

Durieu (1846), Saccardo and Berlese (1886), Kedad and Bouznad (2018).

194. Volutella gilva (Pers.) Sacc. (syn. Psilonia gilva)

Durieu (1846), Kedad and Bouznad (2018).

195. Volutella melaloma Berk. \& Broome

Maire (1917, 1919), Kedad and Bouznad (2018), [BPI], [MA].

\section{Sordariomycetidae}

196. Ambarignomonia petiolorum (Schwein.) Sogonov (syn. Gnomonia petiolorum). Belhoucine et al. (2012), Kedad and Bouznad (2018).

197. Amesia gelasinospora (Arx \& E. Müll.) X.Wei Wang \& Samson Locquin-Linard (1988).

198. Anisogramma vepris (Lacroix) Merezhko (syn. Apioporthe vepris, Diaporthe vepris). Saccardo \& Roumeguère (1881), Kedad and Bouznad (2018)

199. Arthrinium arundinis (Corda) Dyko \& B. Sutton (syn. Apiospora montagnei, Coniosporium arundinis, Sphaeria apiospora, Tubercularia apiospora).

Durieu (1846), Montagne (1856), Saccardo and Roumeguère (1881), Saccardo (1882, 
1886), Saccardo and Berlese (1886), Maire (1906, 1917), Kedad and Bouznad (2018), [BPI], [MA], [MPU], [P].

200. Arthrinium phaeospermum (Corda) M.B. Ellis (syn. Coniosporium inquinans, Melanconium sphaerospermum).

Durieu (1846), Montagne (1856), Saccardo (1886), Bensaci et al. (2015), Kedad and Bouznad (2018), [P]

201. Asteroma ceratoniae Sacc.

[BR], [MIN].

202. Asteroma concinnum Durieu \& Mont.

Montagne (1856), Saccardo (1884), Roumeguère (1886), Kedad and Bouznad (2018), [MIN], [BR], [P].

203. Asteroma guepinii Mont.

Montagne (1857).

204. Asteroma medusula Durieu \& Mont.

Montagne (1856), Saccardo (1884), Maire and Werner (1938), Kedad and Bouznad (2018), [BR], [MIN], [P].

205. Asteroma rhoinum Durieu \& Mont. Montagne (1856), Saccardo (1884), Kedad and Bouznad (2018), [P].

206. Asteroma tenerrimum Grognot

Maire and Werner (1938), Kedad and Bouznad (2018).

207. Asteroma venulosum (Wallr.) Fuckel

Maire and Werner (1938), Kedad and Bouznad (2018).

208. Bombardia musae (Maire) C. Moreau (syn. Lasiosordaria musae, Podospora musae) Maire (1917), Kedad and Bouznad (2018), [MPU].

209. Botryodiplodia aesculina Pass.

[H], [MPU].

210. Botryotrichum spirotrichum (R.K. Benj.) X.Wei Wang \& Samson (syn. Chaetomidium spirotrichum).

Locquin-Linard (1988), [P].

211. Camptosphaeria clavispora (S.I. Ahmed \& J.H. Mirza) J.C. Krug \& Jeng (syn. Podospora cf clavispora).

Locquin-Linard (1988).

212. Ceratostomella leptorrhyncha (Durieu \& Mont.) Sacc.

Saccardo (1882), Kedad and Bouznad (2018).

213. Cercophora ambigua (Sacc.) R. Hilber (syn. Lasiosphaeria ambigua) Saccardo (1883).

214. Cercophora coprophila (Fr.) N. Lundq. (syn. Bombardia coprophila). Faurel \& Schotter (1964a). 
215. Chaetomidium cephalothecoides (Malloch \& Benny) Arx (syn. Chaetomidium cephalotecoides)

Locquin-Linard (1988).

216. Chaetomidium cylindraceum

Locquin-Linard (1988).

217. Chaetomidium ovalisporum (Lodha) Lodha (syn. Thielavia ovalispora)

Locquin-Linard (1988).

218. Chaetomium brevipilium L.M. Ames

Locquin-Linard (1988).

219. Chaetomium caprinum Bainier

Locquin-Linard (1988), [P].

220. Chaetomium cochliodes Palliser

Kahoul et al. (2014).

221. Chaetomium elatum Kunze

Durieu (1846), Dubost (1966), Kedad and Bouznad (2018).

222. Chaetomium globosum Kunze (syn. C. globosum var. ochraceoides, C. olivaceum). Nicot (1955), Faurel and Schotter (1964a, b), Dubost (1966), Locquin-Linard (1988), Belhoucine et al. (2012), Zamoum et al. (2011), Kedad and Bouznad (2018).

223. Chaetomium jodhpurense Lodha Locquin-Linard (1988).

224. Chaetomium madrasense Natarajan (syn. Chaetomium ascotrichoides)

Locquin-Linard (1988), [P].

225. Chaetomium mareoticum Besada \& Yusef

Locquin-Linard (1988), [P].

226. Chaetomium mollipilium L.M. Ames

Locquin-Linard (1988).

227. Chaetomium murorum Corda (syn. Botryotrichum murorum).

Durieu (1846), Dubost (1966), Locquin-Linard (1988), Kedad and Bouznad (2018),

Chamekh et al. (2019), [P].

228. Chaetomium perlucidum Sergeeva

Locquin-Linard (1988).

229. Chaetomium piluliferum J. Daniels (syn. Botryotrichum piluliferum).

Locquin-Linard (1988), Bekhouche et al. (1994), Chamekh et al. (2019).

230. Chaetomium retardatum A. Carter \& R.S. Khan

Chamekh et al. (2019).

231. Chaetomium spirale Zopf

Faurel and Schotter (1964b), Locquin-Linard (1988). 
232. Chaetomium spiralotrichum Lodha

$[\mathrm{P}]$.

233. Chaetomium strumarium (J.N. Rai, J.P. Tewari \& Mukerji) P.F. Cannon Locquin-Linard (1988).

234. Collariella bostrychodes (Zopf) X.Wei Wang \& Samson (syn. Chaetomium bostrychodes).

Faurel and Schotter (1964a), Locquin-Linard (1988).

235. Collariella quadrangulata (Chivers) X.Wei Wang \& Samson Locquin-Linard (1988).

236. Coniochaeta ligniaria (Grev.) Cooke

Maire (1906), Locquin-Linard (1988), Kedad and Bouznad (2018), [P].

237. Coniochaeta lignicola (Nannf.) Z.U. Khan, Gené \& Guarro (syn. Lecythophora lignicola).

$[\mathrm{P}]$.

238. Coniochaeta pulveracea (Ehrh.) Munk (syn. Rosellinia pulveracea).

Maire (1906), Kedad and Bouznad (2018).

239. Coniochaeta sordaria (Fr.) Petr. (syn. Sphaeria sordaria).

Durieu (1846), Kedad and Bouznad (2018), [P].

240. Coryneum microstictoides Sacc. \& Penz.

[MPU].

241. Coryneum microstictum Berk. \& Broome

Saccardo and Roumeguère (1881), Saccardo (1884), Kedad and Bouznad (2018).

242. Cytospora ceratosperma (Tode) G.C. Adams \& Rossman

Saccardo and Roumeguère (1881), Kedad and Bouznad (2018).

243. Cytospora cryptostoma Durieu \& Mont.

$[\mathrm{P}]$.

244. Cytospora draconis Sacc. \& Berl.

Saccardo and Berlese (1886), Saccardo (1886, 1892), Kedad and Bouznad (2018).

245. Cytospora libertella Durieu \& Mont.

Montagne (1856), Saccardo (1884), Kedad and Bouznad (2018), [BR], [P].

246. Cytospora paradoxa (Durieu \& Mont.) Sacc.

Saccardo (1884), Kedad and Bouznad (2018).

247. Cytospora populina (Pers.) Rabenh. (syn. Sphaeria microstoma).

Durieu (1846), Saccardo and Berlese (1886), Kedad and Bouznad (2018), [P].

248. Cytospora punicae Sacc.

Saccardo \& Berlese (1886), Kedad and Bouznad (2018).

249. Cytospora salicis (Corda) Rabenh.

Saccardo and Berlese (1886), Kedad and Bouznad (2018). 
250. Cytospora salsolae Durieu \& Mont.

$[\mathrm{P}]$.

251. Depazea vagans (Fr.) Fr. (syn. Sphaeria vagans).

Steinheil (1834), Boivin (1840).

252. Dichotomopilus indicus (Corda) X.Wei Wang \& Samson (syn. Chaetomium indicum). Locquin-Linard (1988).

253. Dictyoarthrinium sacchari (J.A. Stev.) Damon

Locquin-Linard (1988).

254. Diplodina cynarae Kill. \& Maire

Killian and Maire (1927, 1928), Kedad and Bouznad (2018), [MPU].

255. Gaeumannomyces graminis (Sacc.) Arx \& D.L. Olivier (syn. Ophiobolus cariceti, Ophiobolus graminis).

[BPI], [CUP].

256. Gaeumannomyces tritici (J. Walker) Hern.-Restr. \& Crous (syn. G. graminis var. tritici).

[BPI].

257. Harknessia uromycoides (Speg.) Speg.

Maire (1919), Kedad and Bouznad (2018), [BPI], [MA].

258. Humicola fuscoatra Traaen

Locquin-Linard (1988).

259. Hyalocylindrophora rosea (Petch) Réblová \& W. Gams (syn. Acremonium roseum) [Herbarium G], [MPU].

260. Jattaea algeriensis Berl.

Saccardo (1902), Réblová (2011), Wijayawardene et al. (2017), Kedad and Bouznad (2018).

261. Jattaea spermatozoides Berl.

Kedad and Bouznad (2018).

262. Laestadia bupleuri (Durieu \& Mont.) Sacc.

Saccardo (1882), Kedad and Bouznad (2018)

263. Lasiosphaeria montagnei (Fr. ex Mont.) Sacc.

Durieu (1846), Montagne (1856), Saccardo (1883), Kedad and Bouznad (2018), [P].

264. Leucostoma persoonii (Nitschke) Höhn. (syn. Sphaeria leucostoma).

$[\mathrm{P}]$.

265. Linospora saligna (Ehrh. ex Pers.) Traverso (syn. Sphaeria saligna).

Durieu (1846), Kedad and Bouznad (2018).

266. Madurella clapieri (Catanei) Redaelli \& Cif. (syn. Glenospora clapieri)

Montpelier and Catanei (1927), Catanei and Legroux (1931), Catanei (1938). 
267. Madurella mycetomatis (Laveran) Brumpt

Montpelier and Catanei (1927), Catanei and Legroux (1931), Catanei (1938), Tligui et al. (2006), Zait et al. (2008).

268. Mazzantia gougetiana (Mont.) Mont. (syn. Dothidea gougetiana). Montagne (1842), Saccardo (1883), Kedad and Bouznad (2018), [S].

269. Melanconium ammophilae (Durieu \& Mont.) Höhn. (syn. Cryptosporium ammophilae).

Durieu (1846), Montagne (1856), Kedad and Bouznad (2018), [P].

270. Melanconium disseminatum Link

Durieu (1846), Kedad and Bouznad (2018).

271. Melanconium eriobotryae Maire Maire (1945), Kedad and Bouznad (2018), CMI (1954).

272. Neurospora sitophila Shear \& B.O. Dodge (syn. Chrysonilia sitophila). Belhoucine et al. (2012).

273. Ophiognomonia leptostyla (Fr.) Sogonov Kedad and Bouznad (2018).

274. Ophiognomonia setacea (Pers.) Sogonov (syn. Sphaeria setacea). Durieu (1846), Kedad and Bouznad (2018)

275. Ovatospora brasiliensis (Bat. \& Pontual) X.Wei Wang \& Samson (syn. Chaetomium brasiliense). Locquin-Linard (1988), [P].

276. Pesotum piceae J.L. Crane \& Schokn. (syn. Ophiostoma quercus). Belhoucine et al. (2012), Kedad and Bouznad (2018).

277. Phaeoacremonium hispanicum Gramaje, Armengol \& L. Mostert Berraf-Tebbal et al. (2011), Larignon (2016), Kedad and Bouznad (2018).

278. Phaeoacremonium inflatipes W. Gams, Crous \& M.J. Wingf. Belhoucine et al. (2012), Kedad and Bouznad (2018).

279. Phaeoacremonium leptorrhynchum (Durieu \& Mont.) D. Gramaje, L. Mostert \& Crous (syn. Sphaeria leptorhyncha, Togninia leptorrhyncha). Durieu (1846), Montagne (1856), Réblová (2011), [P].

280. Phaeoacremonium minimum (Tul. \& C. Tul.) Gramaje, L. Mostert \& Crous (syn. P. aleophilum).

Berraf and Péros (2005), Berraf-Tebbal et al. (2011), Larignon (2016), Kedad and Bouznad (2018).

281. Phaeoacremonium parasiticum (Ajello, Georg \& C.J.K. Wang) W. Gams, Crous \& M.J. Wingf.

Berraf-Tebbal et al. (2011), Larignon (2016), Kedad and Bouznad (2018).

282. Phaeoacremonium venezuelense $L$. Mostert, Summerb. \& Crous Berraf-Tebbal et al. (2011), Larignon (2016), Kedad and Bouznad (2018). 
283. Phomopsis acanthi (Sacc. \& D. Sacc.) Grove (syn. Phoma acanthi).

[MPU].

284. Phomopsis achilleae (Sacc.) Traverso (syn. Diaporthe orthoceras). Maire (1919), Kedad and Bouznad (2018), [BPI], [MPU].

285. Phomopsis algerica Maire

Maire (1945), Kedad and Bouznad (2018), CMI (1954).

286. Phomopsis arctii (Lasch) Traverso (syn. Sphaeria inquilina).

Durieu (1846), Kedad and Bouznad (2018).

287. Phomopsis cycadis (Sacc. \& Berl.) M.T. Lucas \& Sousa da Câmara (syn. Phoma cycadis).

Saccardo and Berlese (1886), Saccardo (1886, 1892), Kedad and Bouznad (2018).

288. Phomopsis ferulae [MPU].

289. Phomopsis inaequalis Traverso (syn. Diaporthe inaequalis). Saccardo and Roumeguère (1881), Kedad and Bouznad (2018)

290. Phomopsis lathyrina (Sacc.) Grove (syn. Phoma lathyrina). Saccardo and Berlese (1886), Kedad and Bouznad (2018).

291. Phomopsis marrubii (Durieu \& Mont.) Grove (syn. Phoma marrubii, Sphaeronaema marrubii).

Durieu (1846), Saccardo (1884), Maire and Werner (1938), Kedad and Bouznad (2018).

292. Phomopsis piceae (Pers.) Höhn. (syn. Diaporthe picea, Euporthe picea, Sphaeria picea).

Durieu (1846), Saccardo (1882), Maire (1916), Kedad and Bouznad (2018), [BPI], [P].

293. Phomopsis prunorum (Cooke) Grove (syn. Phomopsis mali).

Dorléans and Emmanouilidis (1972).

294. Phomopsis pulla (Sacc.) Traverso (syn. Diaporthe pulla).

Maire (1917), Kedad and Bouznad (2018), [BPI].

295. Phomopsis sarothamni Höhn. (syn. Phoma sarothamni).

[ILL].

296. Phomopsis scabella (Penz.) Curzi (syn. Phoma scabella). [MPU].

297. Phomopsis striiformis (Durieu \& Mont.) Grove [as 'striaeformis'] (syn. Phoma striiformis).

Montagne (1856), Saccardo (1884), Roumeguère (1885), Stevenson (1926), Kedad and Bouznad (2018).

298. Phomopsis stromatigena Maire

Maire (1917), Kedad and Bouznad (2018), [MPU].

299. Phomopsis velata (Sacc.) Traverso (syn. Sphaeria discutiens).

Durieu (1846), Kedad and Bouznad (2018), [P]. 
300. Phomopsis vexans (Sacc. \& P. Syd.) Harter (syn. Ascochyta hortorum var. compositarum).

Maire and Werner (1938), Kedad and Bouznad (2018).

301. Phomopsis vicina (Desm.) Grove

Kedad and Bouznad (2018).

302. Podospora decipiens (G. Winter) Niessl (syn. Pleurage decipiens).

Faurel and Schotter (1964a), Richardson (2004).

303. Podospora mexicana Mirza \& Cain

Locquin-Linard (1988).

304. Podospora minicauda Faurel \& Locq.-Lin. Locquin-Linard (1978, 1988), CMI (1981).

305. Podospora pleiospora (G. Winter) Niessl (syn. Pleurage decipiens f. pleiospora). Faurel and Schotter (1964a).

306. Podospora prethopodalis Cain Locquin-Linard (1988).

307. Podospora seminuda (Griffiths) Mirza \& Cain Locquin-Linard (1988).

308. Podospora setosa (G. Winter) Niessl (syn. Pleurage setosa). Faurel and Schotter (1964a), Locquin-Linard (1988).

309. Raffaelea montetyi M. Morelet Belhoucine et al. (2012), Kedad and Bouznad (2018).

310. Schizothecium conicum (Fuckel) N. Lundq. (syn. Pleurage curvula). Faurel and Schotter (1964a).

311. Schizothecium dubium (E.C. Hansen) N. Lundq. (syn. Podospora dubia). Locquin-Linard (1988).

312. Schizothecium fimbriatum (A. Bayer) Barrasa \& Soláns (syn. Podospora fimbriata). Locquin-Linard (1988).

313. Schizothecium tetrasporum (G. Winter) N. Lundq. (syn. Pleurage minuta f. tetraspora, Podospora tetraspora). Faurel and Schotter (1964a), Locquin-Linard (1988), Richardson (2004).

314. Schizothecium vesticola (Berk. \& Broome) N. Lundq. Richardson (2004).

315. Sporothrix schenckii Hektoen \& C.F. Perkins (syn. Rhinocladium beurmannii). Catanei $(1934,1938)$.

316. Sporothrix stenoceras (Robak) Z.W. de Beer, T.A. Duong \& M.J. Wingf. (syn. Ophiostoma stenoceras).

Belhoucine et al. (2012), Kedad and Bouznad (2018). 
317. Subramaniula cuniculorum (Fuckel) X.Wei Wang \& Samson (syn. Chaetomium cuniculorum).

Locquin-Linard (1988).

318. Thielavia basicola Zopf

[P].

319. Thielavia bispora Lodha

Locquin-Linard (1988).

320. Thielavia coactilis Nicot

Locquin-Linard (1988), [P].

321. Thielavia coprophila (R.K. Pathak \& S.C. Agarwal) D. Hawksw.

Locquin-Linard (1988).

322. Thielavia microspora Mouch.

Locquin-Linard (1988), Bekhouche et al. (1994).

323. Thielavia terricola (J.C. Gilman \& E.V. Abbott) C.W. Emmons Locquin-Linard (1988), [P].

324. Trichocladium asperum Harz Kedad and Bouznad (2018).

325. Trichocladium griseum (Traaen) X. Wei Wang \& Houbraken (syn. Humicola grisea). Locquin-Linard (1988), Kedad and Bouznad (2018).

326. Valsa campsospora (Mont.) Mont. (syn. Sphaeria campsospora). Durieu (1846), Saccardo (1882), Kedad and Bouznad (2018).

327. Valsa crocina (Durieu \& Mont.) Mont. (syn. Sphaeria crocina). Durieu (1846), Montagne (1856), Saccardo (1882), Kedad and Bouznad (2018), [P].

328. Valsa mendax (Mont.) Mont. (syn. Sphaeria mendax).

Durieu (1846), [BPI], [P].

329. Valsa mendax (Mont.) Mont. Montagne (1856), Saccardo (1882), Kedad and Bouznad (2018).

330. Valsa praeclara (Mont.) Sacc. (syn. Sphaeria praeclara).

Durieu (1846), Saccardo (1882), [P].

331. Valsa praeclara (Mont.) Sacc.

Montagne (1856), Kedad and Bouznad (2018).

332. Valsa sordida Nitschke (syn. Cytispora chrysosperma). Saccardo and Berlese (1886), Maire and Werner (1938), Kedad and Bouznad (2018).

333. Valsaria donacina (De Not.) De Not. (syn. Sphaeria donacina). Saccardo (1882).

334. Valsaria insitiva (Tode) Ces. \& De Not. (syn. Eutypa viticola, Sphaeria viticola). Saccardo (1882). 
335. Zopfiella erostrata (Griffiths) Udagawa \& Furuya (syn. Tripterospora erostrata). Faurel and Schotter (1964b), Locquin-Linard (1988).

336. Zygopleurage multicaudata Mirza

Locquin-Linard (1988).

337. Zygopleurage zygospora (Speg.) Boedijn (syn. Pleurage zygospora).

Faurel and Schotter (1964a).

\section{Xylariomycetidae}

338. Amphisphaerella xylostei (Pers.) Rulamort (syn. Anthostoma xylostei, Sphaeria xylostei).

Durieu (1846), Saccardo (1882), Kedad and Bouznad (2018).

339. Amphisphaeria dichroa (Durieu \& Mont.) Ces. \& De Not.

Durieu (1846), Montagne (1856), Saccardo (1882), Kedad and Bouznad (2018), [P].

340. Amphisphaeria diplasia (Durieu \& Mont.) Sacc.

Durieu (1846), Montagne (1856), Saccardo (1882), Kedad and Bouznad (2018), [P].

341. Amphisphaeria leptosphaerioides Maire

[G], [MPU].

342. Amphisphaeria umbrina (Fr.) De Not. (syn. A. conica)

Saccardo (1882), Maire (1906), Kedad and Bouznad (2018).

343. Annulohypoxylon truncatum (Starbäck) Y.M. Ju, J.D. Rogers \& H.M. Hsieh (syn. Sphaeria depressa).

[BPI], [P].

344. Anthostoma defossum (Durieu \& Mont.) Sacc. (syn. Sphaeria defossa).

Durieu (1846), Montagne (1856), Saccardo (1882), Kedad and Bouznad (2018).

345. Anthostoma gastrinum (Fr.) Sacc.

Saccardo and Berlese (1886), Patouillard (1903), Kedad and Bouznad (2018).

346. Anthostoma phillyreae (Mont.) Sacc. (syn. Diatrype phillyreae). Montagne (1856), Saccardo (1882), Kedad and Bouznad (2018).

347. Anthostoma sicyosperma (Durieu \& Mont.) Sacc. (syn. Sphaeria sicyosperma). Durieu (1846), Montagne (1856), Saccardo (1882), Kedad and Bouznad (2018), [P].

348. Anthostomella acanthina (Durieu \& Mont.) Sacc. (syn. Sphaeria acanthina). Durieu (1846), Montagne (1856), Saccardo (1882), Kedad and Bouznad (2018), [P].

349. Anthostomella appendiculosa (Berk. \& Broome) Sacc. Maire (1917), Kedad and Bouznad (2018), [Herbarium G], [MPU].

350. Anthostomella chionostoma (Durieu \& Mont.) Sacc. (syn. Sphaeria chionostoma). Durieu (1846), Montagne (1856), Saccardo (1882), Kedad and Bouznad (2018), [P].

351. Anthostomella contaminans (Durieu \& Mont.) Sacc. (syn. Sphaeria contaminans, S. phoenicis). 
Durieu (1846), Montagne (1856), Saccardo (1882), Frohlich (1997), Kedad and Bouznad (2018), [BR], [MIN], [P].

352. Anthostomella duplex (Sowerby) Sacc. (syn. Sphaeria duplex var. rottboelliae). Steinheil (1834), Boivin (1840).

353. Anthostomella intybi (Durieu \& Mont.) Sacc. (syn. Sphaeria intybi).

Durieu (1846), Montagne (1856), Saccardo (1882), Kedad and Bouznad (2018), [P].

354. Anthostomella nitidissima (Durieu \& Mont.) Sacc. (syn. Sphaeria nitidissima).

Durieu (1846), Montagne (1856), Saccardo (1882), Kedad and Bouznad (2018), [P].

355. Anthostomella phoenicicola Speg.

Dennis (1984).

356. Anthostomella scotina (Durieu \& Mont.) Sacc. (syn. Sphaeria scotina).

Durieu (1846), Montagne (1856), Saccardo (1882), Kedad and Bouznad (2018), [P].

357. Anthostomella trabutiana Sacc. \& Roum.

Saccardo and Roumeguère (1881), Saccardo (1882), Kedad and Bouznad (2018).

358. Biscogniauxia mediterranea (De Not.) Kuntze (syn. Hypoxylon mediterraneum, $H$. sertatum, Sphaeria sertata).

Durieu (1846), Montagne (1856), Saccardo (1882), Maire (1927), Malençon (1952),

Dennis (1984), El-Assfouri et al. (2003), Belhoucine et al. (2012), Kedad and Bouznad (2018), [P].

359. Biscogniauxia phyllireae (Mont.) Rappaz (syn. Sphaeria phyllireae).

Durieu (1846), Kedad and Bouznad (2018), [P].

360. Biscogniauxia repanda (Fr.) Kuntze

[BR]

361. Cainia desmazieri C. Moreau \& E. Müll.

Kedad and Bouznad (2018).

362. Clypeophysalospora latitans (Sacc.) H.J. Swart (syn. Physalospora latitans) [MPU]

363. Clypeosphaeria contempta (Durieu \& Mont.) Sacc.

Durieu (1846), Montagne (1856), Saccardo (1883), Kedad and Bouznad (2018), [P].

364. Clypeosphaeria mamillana (Fr.) Lambotte (syn. Sphaeria clypeata, Sphaeria mamillana).

Durieu (1846), Kedad and Bouznad (2018), [P].

365. Cryptovalsa rabenhorstii (Nitschke) Sacc.

Saccardo and Berlese (1886), Kedad and Bouznad (2018).

366. Daldinia concentrica (Bolton) Ces. \& De Not. (syn. Hypoxylon concentricum, Sphaeria concentrica).

Durieu (1846), Maire (1915), Malençon (1952), El-Assfouri et al. (2003), Youcef-

Khodja 2010, Kedad and Bouznad (2018), [BPI], [P]. 
367. Daldinia fissa Lloyd (syn. Daldinia concentrica var. obovata).

Stadler et al. (2014).

368. Daldinia martinii M. Stadler, Venturella \& Wollw.

Stadler et al. (2014).

369. Daldinia vernicosa Ces. \& De Not. (syn. Sphaeria vernicosa).

Durieu (1846), Saccardo (1882), Kedad and Bouznad (2018), [P].

370. Diatrype bullata (Hoffm.) Fr.

Kedad and Bouznad (2018).

371. Diatrype durieui (Mont.) Mont. (syn. Eutypella durieui, Sphaeria durieui).

Durieu (1846), Montagne (1856), Saccardo (1882), Rappaz (1987), Kedad and Bouznad (2018), [BPI], [P].

372. Diatrype leucocreas (Durieu \& Mont.) Mont. (syn. Sphaeria leucocreas).

Durieu (1846), Montagne (1856), Saccardo (1882), Rappaz (1987), Kedad and Bouznad (2018), [P].

373. Diatrype leucostroma (Durieu \& Mont.) Rappaz (syn. Eutypa leucostroma, Sphaeria leucostroma, Valsa leucostroma).

Durieu (1846), Montagne (1856), Saccardo (1882), Rappaz (1987), Kedad and Bouznad (2018).

374. Diatrype stigma (Hoffm.) Fr. (syn. Sphaeria stigma).

Durieu (1846), Maire (1906), Kedad and Bouznad (2018), [P].

375. Diatrypella favacea (Fr.) Ces. \& De Not. (syn. Sphaeria verrucaeformis).

$[\mathrm{P}]$

376. Discosia pleurochaeta Durieu \& Mont.

Durieu (1846), Montagne (1856), Saccardo (1884), Vanev (1996), Kedad and

Bouznad (2018), [P].

377. Dyrithium lividum (Pers.) M.E. Barr (syn. Mycothyridium lividum, Phragmispora livida, Sphaeria livida, Thyridium lividum).

Durieu (1846), Saccardo (1883), Kedad and Bouznad (2018), [P].

378. Euepixylon udum (Pers.) Læssøe \& Spooner (syn. Sphaeria uda).

Durieu (1846), Kedad and Bouznad (2018), [P].

379. Eutypa abscondita (Durieu \& Mont.) Rappaz (syn. Eutypa lata var. abscondita). Saccardo (1882), Rappaz (1987).

380. Eutypa consobrina (Mont.) Rappaz (syn. Diatrype consobrina, Sphaeria consobrina). Durieu (1846), Montagne (1856), Saccardo (1882), Rappaz (1987), Kedad and Bouznad (2018), [P].

381. Eutypa lata (Pers.) Tul. \& C. Tul. (syn. Sphaeria lata, S. milliaria).

Durieu (1846), Saccardo (1882), Patouillard (1897a), Berraf and Péros (2005), Ammad et al. (2014), Kedad and Bouznad (2018), [P]. 
382. Eutypa leptoplaca (Durieu \& Mont.) Rappaz

Kedad and Bouznad (2018).

383. Eutypa orthosticha (Durieu \& Mont.) Rappaz (syn. Diatrype orthosticta, Sphaeria orthosticha).

Durieu (1846), Montagne (1856), Saccardo (1882), Rappaz (1987), Kedad and Bouznad (2018), [P].

384. Eutypa sycina (Durieu \& Mont.) Sacc. (syn. Sphaeria sycina, Valsa salicina). Durieu (1846), Montagne (1856), Saccardo (1882), Kedad and Bouznad (2018), [P].

385. Eutypa tetragona (Duby) Sacc. (syn. Sphaeria tetragona). Durieu (1846), Saccardo (1882), Kedad and Bouznad (2018).

386. Eutypella atropae (Durieu \& Mont.) Sacc. (syn. Sphaeria atropae, Valsa atropae). Durieu (1846), Montagne (1856), Saccardo (1882), Rappaz (1987), Kedad and Bouznad (2018), [P].

387. Eutypella cheirolophi Maire Maire (1917, 1919), Kedad and Bouznad (2018), [BPI], [MA], [MPU].

388. Eutypella exanthemoides (Mont.) Sacc. (syn. Sphaeria exanthemoides, Valsa exantiiemoides).

Durieu (1846), Montagne (1856), Saccardo (1882), Rappaz (1987), Kedad and Bouznad (2018), [P].

389. Eutypella prunastri (Pers.) Sacc. Maire (1917), Kedad and Bouznad (2018), [BPI], [MA].

390. Eutypella stellulata (Fr.) Sacc. (syn. Sphaeria stellulata). Durieu (1846), Kedad \& Bouznad (2018), [P].

391. Hyaloceras notarisii Durieu \& Mont. Saccardo (1884).

392. Hypocopra lasioderma (Durieu \& Mont.) Sacc. (syn. Sphaeria lasioderma). Durieu (1846), Montagne (1856), Saccardo (1882), Kedad and Bouznad (2018), [P].

393. Hypocopra stercoraria (Sowerby) Sacc. (syn. Sphaeria stercoraria var. macrospora). Durieu (1846), Montagne (1856), Saccardo (1882), Kedad and Bouznad (2018), [P].

394. Hypocrea citrina De Not. [BPI]

395. Hypocrea gracilis Durieu \& Mont.

$[\mathrm{P}]$

396. Hyponectria cooperta (Desm.) M.E. Barr (syn. Laestadia cooperta). [MPU]

397. Hyponectria jucunda (Mont.) Weese (syn. Hypocrea jucunda, Nectrlella jucunda). Montagne (1856), Saccardo (1878 \& 1883), Kedad \& Bouznad (2018), [P]. 
398. Hypoxylon cornutum var. cupressiforme (With.) Gray (syn. Clavaria cupressiforme, Hypoxylon cornutum var. cupressiforme).

$[\mathrm{P}]$

399. Hypoxylon fuscum (Pers.) Fr. (syn. Sphaeria fusca).

Durieu (1846), Patouillard (1897a,b), Kedad and Bouznad (2018), [P].

400. Hypoxylon rubiginosum (Pers.) Fr. (syn. Sphaeria rubiginosa). Durieu (1846), Saccardo (1882), Kedad and Bouznad (2018), [P].

401. Hypoxylon sublimbatum (Durieu \& Mont.) P.M.D. Martin (syn. Rosellinia sublimbata, Sphaeria sublimbata).

Durieu (1846), Montagne (1856), Saccardo (1882), Kedad and Bouznad (2018), [BPI], $[\mathrm{P}]$.

402. Jackrogersella multiformis (Fr.) L. Wendt, Kuhnert \& M. Stadler (syn.

Annulohypoxylon multiforme).

Durieu (1846), Kedad and Bouznad (2018), [P].

403. Kretzschmaria deusta (Hoffm.) P.M.D. Martin (syn. Ustulina deusta).

Maire (1906), Kedad and Bouznad (2018).

404. Lopadostoma linospermum (Durieu \& Mont.) Jaklitsch, J. Fourn. \& Voglmayr (syn. Anthostoma linospermum, Diatrype linosperma, Sphaeria linosperma).

Durieu (1846), Montagne (1856), Saccardo (1882), Kedad and Bouznad (2018).

405. Massaria rhyponta (Durieu \& Mont.) Mont. (syn. Sphaeria rhyponta).

Durieu (1846), Montagne (1856), Saccardo (1883), Kedad and Bouznad (2018), [P].

406. Melogramma henriquesii Briosi \& Cavara

Saccardo (1913), [S].

407. Microdochium nivale (Fr.) Samuels \& I.C. Hallett (syn. Fusarium nivale).

Dubost (1966), Kedad and Bouznad (2018).

408. Microdochium panattonianum (Berl.) B. Sutton, Galea \& T.V. Price

Kedad and Bouznad (2018).

409. Monochaetia bicornis (Durieu \& Mont.) Sacc. \& D. Sacc. (syn. M. mucronata, Pestalotia bicornis).

Durieu (1846), Montagne (1856), Saccardo (1884), Maire (1906), Kedad and Bouznad (2018), [BPI], [P].

410. Nodulisporium didymosporum Nicot

$[\mathrm{P}]$

411. Penzigia mauritanica (Durieu \& Mont.) J.H. Mill. (syn. Hypoxylon mauritanicum, H. biocellatum, Kretzschmaria mauritanica, Sphaeria mauritanica).

Durieu (1846), Montagne (1856), Saccardo (1882), Patouillard (1902), Frohlich (1997), Kedad and Bouznad (2018), [BPI], [P].

412. Peroneutypa alsophila (Durieu \& Mont.) Carmarán \& A.I. Romero (syn. Eutypa alsophila., Eutypella alsophila, Sphaeria alsophila). 
Durieu (1846), Montagne (1856), Saccardo (1882), Rappaz (1987), Kedad and Bouznad (2018), [P].

413. Peroneutypa bellula (Desm.) Berl. (syn. Sphaeria bellula).

Durieu (1846), Kedad and Bouznad (2018), [P].

414. Peroneutypa scoparia (Schwein.) Carmarán \& A.I. Romero (syn. Eutypa heteracantha).

Saccardo and Roumeguère (1881), Kedad and Bouznad (2018).

415. Pestalotia algeriensis (Sacc. \& Berl.) Guba (syn. P. funerea var. algeriensis, $P$. funerea var. populi, Pestalotiopsis algeriensis).

Saccardo (1886, 1892), Saccardo and Berlese (1886), Kedad and Bouznad (2018), [BPI].

416. Pestalotia rosae Westend.

Patouillard (1903), Kedad and Bouznad (2018).

417. Pestalotiopsis breviseta (Sacc.) Steyaert (syn. Pestalotia breviseta).

Saccardo and Berlese (1886), Kedad and Bouznad (2018).

418. Pestalotiopsis decolorata (Speg.) Steyaert (syn. Pestalotia decolorata).

Roumeguère (1887), [BR].

419. Pestalotiopsis guepinii (Desm.) Steyaert (syn. Pestalotia guepini).

Durieu (1846), Kedad and Bouznad (2018), [P].

420. Pestalotiopsis paraguariensis Y.X. Chen (syn. Pestalotia paraguayensis).

Mouchacca and Saint-Jore (2000).

421. Pestalotiopsis versicolor (Speg.) Steyaert

Locquin-Linard (1988), [P].

422. Physalospora ventricosa Durieu \& Mont. ex Cooke

Saccardo (1895).

423. Pontoporeia biturbinata (Durieu \& Mont.) Kohlm. (syn. Amphisphaeria biturbinata, Sphaeria biturbinata).

Durieu (1846), Montagne (1856), Saccardo (1882), Kohlmeyer (1963), Kedad and Bouznad (2018), [P].

424. Quaternaria persoonii Tul. \& C. Tul. [MPU]

425. Rosellinia aquila (Fr.) Ces. \& De Not. (syn. Sphaeria aquila).

Durieu (1846), Maire (1919), Kedad \& Bouznad (2018), [BPI], [P].

426. Rosellinia verrucaria Mont. ex Sacc.

Saccardo (1882).

427. Sarcostroma bisetulatum Guba ex Nag Raj (syn. Cryptostictis bisetulata). CMI (1972).

428. Seiridium cardinale (W.W. Wagener) B. Sutton \& I.A.S. Gibson Bonthond et al. (2018). 
429. Sphaeria bromeliae Schwein.

[G], [BR], [BRLU].

430. Sphaeria crucianellae Durieu \& Mont.

Durieu (1846), Montagne (1856), [P].

431. Sphaeria culmicola Fries var. arundinis

[P]

432. Sphaeria dispersa Mont.

Boivin (1840).

433. Sphaeria inquilina $\mathrm{Fr}$.

[P]

434. Sphaeria irideas Cesati

[P]

435. Sphaeria jucunda Durieu \& Mont.

Durieu (1846), [P].

436. Sphaeria leptoplaca Durieu \& Mont.

[P]

437. Sphaeria leveillei Durieu \& Mont.

Durieu (1846), Montagne (1856), [P].

438. Sphaeria minutula Durieu \& Mont.

[P]

439. Sphaeria oreades Fr.

[P]

440. Sphaeria phragmispora Durieu \& Mont.

Durieu (1846), Montagne (1856), Kedad and Bouznad (2018), [BR], [P].

441. Sphaeria relicina Fr.

Durieu (1846), Kedad and Bouznad (2018).

442. Sphaeria relicina var. depilata Durieu \& Mont.

Kedad and Bouznad (2018).

443. Sphaeria saubinetii Durieu \& Mont.

Durieu (1846), [P].

444. Xylaria hypoxylon (L.) Grev. (syn. Xylaria cornuta var. cupressiformis).

Durieu (1846), Maire (1927), Kedad and Bouznad (2018), [P]

445. Xylaria polymorpha (Pers.) Grev.

Adouane (2011).

446. Xylaria putaminum Maire \& Durieu

[MPU]

447. Xylaria scruposa (Fr.) Fr.

[BPI] 
448. Xylaria sicula Pass. \& Beltrani (syn. Thamnomyces siculus).

Maire (1915, 1927), Saccardo (1928), Kedad and Bouznad (2018), [BPI], [MA], [MPU], [MPU].

449. Xylaria trabutii Pat.

Patouillard (1897a), Saccardo (1899), Pfister (1977), Frohlich (1997), Kedad and Bouznad (2018), [FH].

\section{Incertae sedis}

450. Diachora lathyri (Lév.) E. Müll. (syn. Physalospora lathyri, Sphaeria lathyri). Durieu (1846), Montagne (1856), Stevenson (1926), Kedad and Bouznad (2018), [P].

451. Diachora onobrychidis (DC.) Jul. Müll. (syn. Placosphaeria onobrychidis, $P$. onobrychidis var. hedysari). Maire (1919), Kedad and Bouznad (2018), [BPI], [MA], [MPU].

452. Melomastia mastoidea (Fr.) J. Schröt. (syn. Sphaeria mastoidea). Durieu (1846), Maire \& Werner (1938), Kedad and Bouznad (2018).

453. Nigrospora oryzae (Berk. \& Broome) Petch (syn. Nigrospora sphaerica). Dubost (1966).

454. Phaeochora steinheilii (Mont.) E. Müll. (syn. Aplosporella dothideoides, Auerswaldia Chamaeropis, Haplosporella dothideoides, Sordaria palmicola Auersw., Sphaeria steinheilii, Sphaeropsis dothideoides).

Steinheil (1834), Boivin (1840), Durieu (1846), Montagne (1856), Debeaux et al. (1880), Saccardo (1884), Patouillard (1897a), Maire (1906, 1915), Dennis (1984), Frohlich (1997), Kedad and Bouznad (2018), [BPI], [BPI], [MA], [BR], [P], [UPS].

455. Phomatospora berkeleyi Sacc. [MPU]

456. Phomatospora dinemasporium J. Webster (syn. Vermicularia graminum). Durieu (1846), Kedad and Bouznad (2018)

457. Phomatospora minutissima (P. Crouan \& H. Crouan) N. Lundq. (syn. Wallrothiella minutissima).

Faurel and Schotter (1964a, b).

458. Phyllachora bromi Fuckel

Saccardo \& Berlese (1886), Maire (1915), Stevenson (1926), Kedad and Bouznad (2018), [MA].

459. Phyllachora cynodontis Niessl

Maire (1915, 1927), Kedad and Bouznad (2018), [BPI], [MA], [S].

460. Phyllachora cyperi Rehm

Maire (1917), Kedad and Bouznad (2018), [MA].

461. Phyllachora fallax Sacc. (syn. Phyllachora fragosoana).

Maire (1917), Saccardo (1926), Kedad and Bouznad (2018), [MPU]. 
462. Phyllachora graminis (Pers.) Fuckel (syn. Sphaeria graminis).

Durieu (1846), Kedad and Bouznad (2018), [CUP], [MA], [P].

463. Phyllachora sporoboli Pat.

Patouillard (1903), Stevenson (1926), Kedad and Bouznad (2018), [CUP].

464. Polystigma fulvum Pers. ex DC.

Kedad and Bouznad (2018).

465. Polystigma rubrum (Pers.) DC.

Durieu (1846), Maire (1915), Kedad and Bouznad (2018), [BPI], [P].

466. Sordaria fimicola (Roberge ex Desm.) Ces. \& De Not. (syn. Rhizopus fimicola). Locquin-Linard (1988), Kahoul et al. (2014).

467. Sordaria humana (Fuckel) G. Winter

Locquin-Linard (1988).

468. Sordaria superba De Not.

Bordjiba et al. (2009).

469. Stigmatula astragali (Lasch) P.F. Cannon (syn. Physalosporina ducellieri).

Kedad and Bouznad (2018), [MPU].

470. Trabutia quercina (F. Rudolphi ex Fr.) Sacc. \& Roum. (syn. Rhytisma quercinum). Boivin (1840), Durieu (1846), Saccardo and Roumeguère (1881), Saccardo (1882), Patouillard (1897a), Maire (1915), Kedad and Bouznad (2018), [BPI], [MA], [P], [S].

Additional records unsupported by literature and herbarium material

Hypocreomycetidae

471. Ciliciopodium sanguineum Corda (Listed by GBIF)

472. Fracchiaea broomeana (Berk.) Petch (Listed by GBIF)

473. Sarocladium kiliense (Grütz) Summerb. (syn. Acremonium kiliense) (Listed by CABI)

474. Striaticonidium cinctum (Corda) L. Lombard \& Crous (Listed by GBIF)

Meliolomycetidae

475. Meliola littoralis Syd. (Listed by GBIF).

Incertae sedis

476. Cryptomycina filicina (Fr.) L. Holm \& K. Holm (Listed by GBIF).

477. Obryzum corniculatum (Hoffm.) Wallr. (Listed by GBIF). 


\section{References}

Abdelaziz, O., Senoussı, M. M., Oufroukh, A., Birgücü, A. K. and Karaca, İ. 2018. Evaluation of Different Isolates of Entomopathogenic Fungi against Metopolophium dirhodum (Walker) (Homoptera: Aphididea) from Constantine, Algeria. International Journal of Innovative Approaches in Agricultural Research, 2(1): 35-43. doi:10.29329/ijiaar.2018.133.4.

Adouane, M. 2011. Diversité des macromycètes et des mycorhizes du chêne-liège dans deux stations du Nord-Est algérien: une subéraie naturelle et une subéraie envahie par Acacia decurrens (Willd). Master Thesis, Université Badji Mokhtar, Annaba, Algeria, 181 pages.

Amrani, S. and Abdel-Azeem, A. M. 2019. Check-list of Algerian fungi - Part 3: Laboulbeniales (Ascomycota). Microbial Biosystems 4(1):17-30.

Aigoun-Mouhous, W., Elena, G., Cabral, A., León, M., Sabaou, N., Armengol, J., Chaouia, C., Mahamedi, A. E. and Berraf-Tebbal, A. 2019. Characterization and pathogenicity of Cylindrocarpon-like asexual morphs associated with black foot disease in Algerian grapevine nurseries, with the description of Pleiocarpon algeriense sp. nov. European Journal of Plant Pathology, 154(4): 887-901. DOI: 10.1007/s10658-019-01708-z

Ainsworth, G.C. and Austwick, P.K.C. 1959. Fungal Diseases of Animals. Commonwealth Bureau of Animal Health, Review Series N ${ }^{\circ}$.6, Farnham Royal (United Kingdom), 216 pages.

Ammad, F., Benchabane, M. and Toumi, M.A. 2014. Diversity of fungal trunk pathogens associated with grapevine dieback of grapevine in Algeria. Jordan Journal of Biological Sciences, 7(1): $35-39$.

Bekhouche, F., Breton, A. and Gaillard-Martinie B. 1994. Champignons cellulolytiques du sol des zones arides du Sahara algérien - mise en évidence de l'activité cellulasique. Cryptogamie, Mycologie, 15(2): 141-147.

Belhoucine, L., Bouhraoua, R.T., Harrak, M.J. and Samson R. A. 2012. Les champignons associés à Platypus cylindrus (Coleoptera, Platypodidae) dans une subéraie du nord ouest d'Algérie: cas des champignons nuisibles. IOBC/wprs Bulletin, 76: 109-116 109.

Belyagoubi, L. 2006. Effet de quelques essences végétales sur la croissance des moisissures de détérioration des céréales Université Abou-Bekr Belkaid, Tlemcen, Algeria, 76 pages.

Benny. G.L. and Kimbrough. J.W. (1980) A synopsis of the orders and families of Plectomycetes with keys to genera. Mycotaxon, 12(1):1-91.

Bensaci, O.A., Harzallah, D. and Gouaref, K. 2015. Endophytic mycoflora of Cedrus atlantica: diversity patterns and determinism of the phytosanitary situation of Atlas cedar forests in Belezma massif (Algeria). Forest Science and Technology, 11(1): 36-43. DOI: 10.1080/21580103.2014.957352.

Benserradj, O. and Mihoubi, I. (2014) Larvicidal activity of entomopathogenic fungi Metarhizium anisopliae against mosquito larvae in Algeria. Int. J. Curr.Microbiol. App. Sci., 3(1): 54-62.

Benzohra, I.-E.-K. and Megateli, M. 2017. Biological control against Bayoud disease of date palm (Phoenix dactylifera L.) using antagonistic fungi species: Antibios and mycoparasitism studies. International Journal of Science and Research, 6(12): 557-563. DOI: $10.21275 /$ ART20178329. 
Berraf, A. and Péros, J.-P. 2005. Importance de l'eutypiose et de l'esca en algérie et structure de la communauté fongique associée. Journal International des Sciences de la Vigne et du Vin, 39(3): 121-128.

Berraf-Tebbal, A., Bouznad, Z., Santos, J.M., Coelho, M.A., Péros, J.-P. and Phillips, A.J.L. 2011. Phaeoacremonium species associated with Eutypa dieback and esca of grapevines in Algeria. Phytopathologia Mediterranea, 50 (Supplement): S86-S97.

Boivin, L.H. 1840. Enumération des plantes qui croissent spontanément en Barbarie. Revue Scientifique et Industrielle 3: 153-184.

Bonthond, G., Sandoval-Deni,s M., Groenewald, J.Z. and Crous P.W. 2018. Seiridium (Sporocadaceae): an important genus of plant pathogenic fungi. Persoonia, 40:96-118. DOI:10.3767/persoonia.2018.40.04

Bordjiba, O., Bekhouche, F. and Steiman, R. 2009. Biodegradation capability of some species of fungi isolated from contamined soils towards herbicides. Toxicology Letters, 189S: S57-S273. DOI:10.1016/j.toxlet.2009.06.573.

Bourzama, G., Bouderda, K., Meddour, A. and Soumati, B. 2018. Highly heavy metals tolerant fungi isolated from the sand of polluted beaches in the area of Annaba - east of Algeria. Journal of Applied Environmental and Biological Sciences, 8 (7): 1-11.

Boutaiba, S. 1997. Contribution à l'étude de la flore fongique du lac d'El Goléa: taxonomie, écologie et production de métabolites. Master thesis, Université Abou Bekr Belkaid, Tlmcen (Algeria), 76 pages.

CMI: Commonwealth Mycological Institute. 1954. Index of fungi: a list of names of new genera, species and varieties of fungi and lichens, new combinations and new names, compiled from world literature. CMI, Kew, United Kingdom, 431 pages.

CMI: Commonwealth Mycological Institute. 1972. Index of fungi: a list of names of new genera, species and varieties of fungi and lichens, new combinations and new names, compiled from world literature. CMI, Kew, United Kingdom, 569 pages.

CMI: Commonwealth Mycological Institute. 1981. Index of fungi: a list of names of new genera, species and varieties of fungi and lichens, new combinations and new names, compiled from world literature. CMI, Kew, United Kingdom, 643 pages.

Cai, L., Hyde, K.D. and Tsui, C. K. M. 2006. Genera of freshwater fungi. Fungal Divers Res Ser 18:1-261.

Debbab, A., Aly, A.H. and Proksch, P. 2013. Mangrove derived fungal endo-phytes-a chemical and biological perception. Fungal Divers 61:1-27

Catanei, A. 1934. Étude parasitologique de trois mycétomes du pied observés en Algérie en 1933. Archives de l'Institut Pasteur d'Algérie, 12: 169-180.

Catanei, A. 1938. La flore parasitaire des mycoses de l'homme en Algérie. Archives de l'Institut Pasteur d'Algérie, $16: 18-20$.

Catanei, A. and Legroux C. 1931. Un nouveau cas de mycétome observé en Algérie. Archives de l'Institut Pasteur d'Algérie, 9(1): 378-381.

Chafai, D. 1996. Micromycètes des sédiments d'oueds et d'effluents industriels de l'Est algérien. Th. Doctorat, Univ. Joseph Fourier, Grenoble, France, 145 p.

Chamekh, R., Deniel, F., Donot, C., Jany, J-L., Nodet, P. and Belabid, L. 2019. Isolation, Identification and enzymatic activity of halotolerant and halophilic fungi from the Great Sebkha of Oran in northwestern of Algeria, Mycobiology, 47:2,230-241, DOI: 10.1080/12298093.2019.1623979. 
Chliyeh, M., Touati, J., Selmaoui, K. , Ouazzani Touhami, A. , Filali Maltouf, A., Cherkaoui El Modafar, C., Moukhli, A., Benkirane, R. and Allal Douira, A. 2014. Bibliographic Inventory of the Olive Tree (Olea europaea L.) Fungal Diseases in the World. International Journal of Pure and Applied Bioscience, 2 (3): 46-79.

Debeaux, O., Trabut, A., Therry, J., Combe, A.J. and Roumeguère, C. 1880. Bouquet de champignons nouveaux observés dans le Midi de la France et en Algérie (1879-1880). Revue Mycologique 2(8): 187-191.

Dennis, R. W. G. 1984. Some Ascomycetes from the Maghrib, North Africa. Kew Bulletin, 39(4): 759-770. DOI: $10.2307 / 4107742$.

Dorleans, G. and Emmanouilidis, I. 1972. Présence d'un Coprin dans la Flore d'un Chancre de néflier. Annales de l'Institut national agronomique (Alger), 7: 21-25.

Dubost, D. 1966, Les Champignons des sols salés de l'Ouest Algérien. (1. Caractères généraux). Bulletin de la Société d'Histoire Naturelle de l'Afrique du Nord, 57(1-2): 9-29.

Durieu de Maisonneuve, M.C. 1846. Flore d'Algérie. Cryptogamie, 1ère partie. Imprimerie Impériale, Paris, 600 pages.

Edel-Hermann, V., Gautheron, N. and Steinberg, C. 2011. Genetic diversity of Fusarium oxysporum and related species pathogenic on tomato in Algeria and other Mediterranean countries. Plant Pathology, 61(4): 787-800. DOI : 10.1111/j.13653059.2011.02551.x.

El-Assfouri, A., Ouazzani-Touhami, A., Zidane, L., Fennane, M. and Douira, A. 2003. Inventaire des spécimens fongiques de l'Herbier national de l'Institut Scientifique de Rabat. Bulletin de l'Institut Scientifique, de Rabat, section Sciences de la Vie, 25: 1-23.

Faurel, L. and Schotter, G. 1964a. Notes mycologiques. II. Quelques champignons coprophiles des environs d'Alger. Revue de Mycologie (Paris), 29 (4): 267-283.

Faurel, L. and Schotter, G. 1964b. Notes mycologiques. III. Quelques champignons coprophiles au sud algérois. Revue de Mycologie (Paris), 29(4), 284-295.

Faurel, L. and Schotter, G. 1965. Notes Mycologiques IV. - Champignons coprophiles du Sahara central et notamment de la Tefedest. Revue de Mycologie (Paris), 30(3): 141-165.

Feldmann, J. 1940. Une nouvelle espèce de Sphéropsidée parasite d'une algue marine. Bulletin de la Société d'Histoire Naturelle de l'Afrique du Nord, 31(9):167-170.

Index Fungorum. www.indexfungorum.org. Accessed on $25^{\text {th }}$ of November 2019.

Jagielski, T., Sandoval-Denis, M., Yu, J., Yao, L., Bakula, Z., Kalita, J., Skora, M., Krzysciak, P., de Hoog, G.S., Guarro, J. and Gene, J. 2016. Molecular taxonomy of Scopulariopsislike fungi with description of new clinical and environmental species. Fungal Biol., 120(4): 586-602.

Jaklitsch, W.M. and Voglmayr, H. 2012. Phylogenetic relationships of five genera of Xylariales and Rosasphaeria gen. nov. (Hypocreales). Fungal Diversity 52: $75-$ 98.http://dx.doi.org/10.1007/s13225-011-0104-2.

Jones, E.B.G., Sakayaroj, J., Suetrong, S., Somrithipol, S. and Pang, K.L. 2009. Classification of marine Ascomycota, anamorphic taxa and Basidiomycota. Fungal Divers 35:1-187.

Jones, E.B.G., Suetrong, S., Sakayaroj, J., Bahkali, A.H., Abdel-Wahab, M.A., Boekhout, T. and Pang, K.L. 2015. Classification of marine Ascomycota, Basidiomycota, Blastocladiomycota and Chytridiomycota. Fungal Divers 73:1-72. 
Juel, H.O. 1901: Contributions à la flore mycologique de l'Algérie et de la Tunisie. Bulletin de la Société Mycologique de France, 17(4): 257-273.

Hyde, K.D. and Wong, S.W. 2000. Annulatascus fusiformis sp. nov., a new fresh water ascomycete from the Philippines. Mycologia 92:353-557.

Hyde, K.D., Jones, E.B.G., Liu, J.K., Ariyawansa, H., Boehm, E., Boonmee, S., Braun, U., Chomnunti, P., Crous, P.W., Dai, D.Q., Diederich, P., Dissanayake, A., Doilom, M., Doveri, F., Hongsanan, S., Jayawardena, R., Lawrey, J.D., Li, Y.M., Liu, Y.X., Lücking, R., Monkai, J., Muggia, L., Nelsen, M.P., Pang, K.L., Phookamsak, R., Senanayake, I.C., Shearer, C.A., Suetrong, S., Tanaka, K., Thambugala, K.M., Wijayawardene, N.N., Wikee, S., Wu, H.X., Zhang, Y., Aguirre-Hudson, B., Alias, S.A., Aptroot, A., Bahkali, A.H., Bezerra, J.L., Bhat, D.J., Camporesi, E., Chukeatirote, E., Gueidan, C., Hawksworth, D.L., Hirayama, K., Hoog, S.D., Kang, J.C., Knudsen, K., Li, W.J., Li, X.H., Liu, Z.Y., Mapook, A., McKenzie, E.H.C., Miller, A.N., Mortimer, P.E., Phillips, A.J.L., Raja, H.A., Scheuer, C., Schumm, F., Taylor, J.E., Tian, Q., Tibpromma, S., Wanasinghe, D.N., Wang, Y., Xu, J.C., Yacharoen, S., Yan, J.Y. and Zhang, M. 2013. Families of Dothideomycetes. Fungal Divers 63:1-313.

Hyde, K.D., Nilsson, R.H., Alias, S.A., Ariyawansa, H.A., Blair, J.E., Cai, L., deCock, A.W.A.M., Dissanayake, A.J., Glockling, S.L., Goonasekara, I.D., Gorczak, M., Hahn, M., Jayawardena, R.S., van Kan, J.A.L., Laurence, M.H., Lévesque, C.A., Li, X.H., Liu, J.K., Maharachchikumbura, S.S.N., Manamgoda, D.S., Martin, F.N., Mckenzie, E.H.C., McTaggart, A.R.,,Mortimer, P.E., Nair, P.V.R., Pawlowska, J., Rintoul, T.L., Shivas, R.G.,Spies, C.F.J, Summerell, B.A., Taylor, P.W.J., Terhem, R.B., Udayanga, D., Vaghefi, N., Walther, G., Wilk, M., Wrzosek, M., Xu, J.C., Yan, J.Y. and Zhou, N. 2014 One stop shop: backbones trees for important phytopathogenic genera: I. Fungal Divers 67:21-125.

Kachour, L. 2004. Identification des moisissures isolées à partir des eaux du lac Oubeira (PNEK) et impact des eaux usées sur leur diversité. Master Thesis, Université Badji Mokhtar, Annaba (Algeria), 260 pages.

Kaewchai, S., Soytong, K. and Hyde, K.D. 2009. Mycofungicides and fungalbiofertilizers. Fungal Divers 38:25-50.

Kahoul, M., Amel Alioua, A., Derbal. N. and Ayad, W. 2014, Comportement des micromycètes du sol vis-à-vis de la pollution mercurielle dans la région d'Azzaba. J. Mater. Environ. Sci., 5 (5):1470-147.

Keim, J., Mishra, B., Sharma, R., Ploch, S., Thines. M. 2014. Root-associated fungi of Arabidopsis thaliana and Microthlaspi perfoliatum. Fungal Divers 66:99-111.

Kekad, A. and Bouznad, Z. 2018. Catalogue des champignons d'Algérie. Tarzalt M. Arts graphiques Edition, Alger-Algeria, 334 pages.

Killian, C. and Fehér, D. 1935. Recherches sur les phénomènes microbiologiques des sols sahariens. Annales de l'Institut Pasteur de Paris, 55: 573-622.

Killian, C. and Maire, R. 1927. Une maladie des artichauts de la Mitidja. Bulletin de la Société d'Histoire Naturelle de l'Afrique du Nord, 18(5): 97-98.

Killian, C. and Maire, R. 1928. Sur une nouvelle maladie des artichauts et sur un champignon Diplodina cynarae qui l'accompagne. Bulletin de la Société d'Histoire Naturelle de l'Afrique du Nord, 19(1): 20-23.

Kirk, P.M. and Ansell, A.E. 1992. Authors of Fungal Names. Kew: CAB International, 95 p. 
Kirk, P.M., Cannon, P.F., Minter, D.W. and Stalpers, J.A. 2008. Dictionary of the fungi, 10th edn. CABI, Wallingford.

Kohlmeyer, J. 1963. Répartition de champignons marins (Ascomycètes et Fungi Imperfecti) dans la Méditerranée. Rapports et procés-verbaux des réunions de la Commission internationale pour l'exploration scientifique de la Mer Méditerranée, 17: 723-730.

Lagarde, J. 1913. Champignons in biospeologica. XXXII. Arch. Zool. Expérim. et Gén., 53: 277307.

Lakhdari, W. 2017. Diagnosis of phytopathogenic fungi of Lycopersicon esculentum L. in Oued Righ region (Algerian Sahara). SDRP Journal of Plant Science, 1(1): 22-27.

Laraba, I., Keddad, A., Boureghda H., Abdallah, N., Vaughan, M.M., Proctor, R.H., Busman, M. and O’Donnell, K. 2018. Fusarium algeriense, sp.nov., a novel toxigenic crown rot pathogen of durum wheat from Algeria is nested in the Fusarium burgessii species complex. Mycologia, 109(6): 935-950. DOI: 10.1080/00275514.2018.1425067.

Larignon, P. 2016. Maladies cryptogamiques du bois de la vigne: symptomatologie et agents pathogènes. http://www.vignevin.com, 168 pages.

Lazreg, F. 2015. Importance de la fonte de semis du pin d'Alep (Pinus halepensis Mill.) dans le Nord-Ouest Algérien : Identification morphologique et moléculaire des espèces du genre Fusarium et Globisporangium, pouvoir pathogène et moyens de lutte. Doctorate Thesis, Université Abou Bekr Belkaid, Tlemcen (Algeria), 186 pages.

Lazreg, F., Belabid, L., Sanchez, J., Gallego, E., Garrido-Cardenas, J.A. and Elhaitoum, A. 2013a. First Report of Fusarium acuminatum Causing Damping-Off Disease on Aleppo Pine in Algeria. Plant disease 97 (4), 557-557.

Lazreg, F., Belabid, L., Sánchez, J.C., Gallego, E., Garrido-Cardenas, J.A., and Elhaitoum, A. 2013b. First report of Fusarium redolens as a causal agent of Aleppo pine damping-off in Algeria. Plant disease, 97 (7), 997.

Lazreg, F., Belabid, L., Sánchez, J.C., Gallego, E., Garrido-Cardenas, J.A., and Elhaitoum, A. 2013c. First report of Fusarium chlamydosporum causing damping-off disease on Aleppo pine in Algeria. Plant disease, 97(11): 1506. DOI: 10.1094/PDIS-02-13-0208PDN.

Lazreg, F., Belabid, L., Sánchez, J.C., Gallego, E., Garrido-Cardenas, J.A., and Elhaitoum, A. 2014a. First Report of Fusarium equiseti Causing Damping-Off Disease on Aleppo Pine in Algeria. Plant disease 98 (9), 1268-1268.

Lazreg, F., Belabid, L., Sanchez, J., Gallego, E. and Bayaa, B. 2014b. Pathogenicity of Fusarium spp. associated with diseases of Aleppo-pine seedlings in Algerian forest nurseries. Journal of Forest Science 60 (3), 115-120.

Locquin-Linard, M. 1977. Á propos des genres non ostiolés placés dans la famille des Microascaceae. Revue de Mycologie (Paris), 41: 509-523.

Locquin-Linard, M. 1978. Un nouvel ascomycète coprophile de la famille des Sordariaceae: Podospora minicauda Faurel et Locquin-Linard. Revue de Mycologie (Paris), 42: 341345.

Locquin-Linard, M. 1988. Etude de la mycoflore coprophile des zones arides et semi-arides du nord de l'Afrique. Doctorate thesis, Université Paris VI (France), 244 pages. 
Lumbsch, H.T. and Huhndorf, S.M. 2010. Myconet volume 14. Part one. Outline of ascomycota - 2009. Part two. Notes on Ascomycete systematics. Nos. 4751-5113. Fieldiana Life Earth Science 1: 1-64.http://dx.doi.org/10.3158/1557.1

Lunghini, D. 1978. Primo contributo alla conoscenza di alcuni ifali demaziacei del Sahara algerino, Giornale botanico italiano, 112(5-6): 373-393. DOI: $10.1080 / 11263507809427948$.

Mahiout, D., Bendahmane, B.S. , Benkada, M.Y., Mekouar, H., Berrahal, N. and Rickauer M. 2019. First report of Colletotrichum gloeosporioides on citrus in Algeria. Phytopathologia Mediterranea, 52(2): 355-359. DOI: 10.14601/Phytopathol_Mediterr23223.

Maire, R. 1900. Quelques Urédinées et Ustilaginées nouvelles ou peu connues. Bulletin de la Société Mycologique de France, 16: 65-72.

Maire, R. 1906. Contributions à l'étude de la flore mycologique de l'Afrique du Nord, Bulletin de la Société Botanique de France, 53: CLXXX-CCXV. DOI:10.1080/00378941.1906.10831941.

Maire, R. 1915. Schedae ad Mycothecam boreali-Africanam. Bulletin de la Société d'Histoire Naturelle de l'Afrique du Nord, 6(4,5,8,9): 66-68, 79-84, 127-134, 139-156.

Maire, R. 1916. Schedae ad Mycothecam Boreali-Africanam. Ser. 2, fascs 8-9. Bulletin de la Société d'Histoire Naturelle de l'Afrique du Nord, 7(8): 294-303,

Maire, R. 1917. Schedae ad mycothecam boreali-Africanam. Bulletin de la Société d'Histoire Naturelle de l'Afrique de Nord, 8(4): 74-84. 242-261

Maire, R. 1919. Schedae ad mycothecam boreali-Africanam. Bull. Soc. Hist. Nat. de l'Afrique du Nord 10: 130-151.

Maire, R. 1927. Excursions mycologiques de la Société d'Histoire Naturelle de l'Afrique du Nord dans la forêt de la Réghaia les 18 Novembre 1923 et 23 Novembre 1924. Bulletin de la Société d'Histoire Naturelle de l'Afrique de Nord, 18(5): 121-124.

Maire, R. 1945. Etudes mycologiques - Fascicule 5. Bulletin de la Société d'Histoire Naturelle de l'Afrique du Nord, 36(3): 24-42.

Maire, R. and Werner, R.G. 1937. Fungi Maroccani. Mémoires de la Société des Sciences Naturelles du Maroc, 45: 1-147 (1938).

Malençon, G. 1952. Contribution à l'étude des champignons de la Kroumirie. Bulletin. De la Société Botanique de France, 99: 33-52.

Mohamed-Mahmoud, F., Amine Yekkour, A., Boudffeur, S., Errahmani, M-B. and Krimi, Z. 2016. Root endophytic fungi from date palm (Phoenix dactylifera L.) grove of Algerian Sahara and screening of their growth promotion activities. Advances in Environmental Biology, 10(11): 18-26.

Montagne, C. 1856. Sylloge generum specierumque plantarum cryptogamarum. J.P. Baillière, Paris (France), 114 pages.

Montagne, C. 1857. Huitième centurie de plantes cellulaires nouvelles, tant indigènes qu'exotiques, Décades VI et VII. Annales des Sciences Naturelles, 8: 285-310.

Montpellier, J. and Gouillon, P. 1921. Mycétome du pied (type pied de Madura) dû à l'Aleurisma apiospermum. Bulletin de la Société de Pathologie Exotique, 14(5):285-290.

Montpellier, J. and Catanei, A. 1927. Formes cliniques, histologie pathologique, parasitologie et diagnostic des mycétomes observés en Algérie. Arch. Inst. Pasteur, Algérie 5: 489-518. 
Mouchacca, J. and Saint-Jore V. 2000. Contribution of André Maublanc to mycology. Ii taxonomic status of introduced taxa. Mycotaxon, 74(2) : $301 \cdot 330$.

Mycobank. Www.mycobank.org. Accessed on $25^{\text {th }}$ of November 2019.

Nicot, J. 1955. Un apercu de la microflore fongique des sables désertiques. UNSECO.Arid Zone Research. 5: 95-99.

Patouillard, N.T. 1897a. Additions au catalogue des champignons de la Tunisie. Bulletin de la Société Mycologique de France, 13(4):197-216.

Patouillard, N.T. 1897b. Catalogue Raisonné des Plantes Cellulaires de la Tunisie. Imprimerie Nationale, Paris (France), 158 pages.

Patouillard, N.T. 1902. Description de quelques champignons extra-europeéns. Bulletin de la Société Mycologique de France, 18(4): 300-304.

Patouillard, N.T. 1903. Additions au catalogue des champignons de la Tunisie. Bulletin de la Société Mycologique de France, 19(3):254-261.

PeiGui, L, Doi, Y., Xiang Hua, W. and Qing Bin, W. 2000. The Hypocreaceae of China III. Some fungicolous species of the genus Hypocrea. Mycosystema 19:317-327

Pfister, D.H. 1977. Annotated Index to Fungi Described by N. Patouillard. Contributions of Reed Herbarium, 25: 1-211.

Prados-Rosale, R.C., Roldán-Rodríguez, R., Serena, C., López-Berges, M.S., Guarro, J., Martínez-del-Pozo, A. and Di Pietro, A. 2012. A PR-1-like protein of Fusarium oxysporum functions in virulence on mammalian host. J Biol Chem 287:21970-21979

Pratibha, J., Prabhugaonkar, A., Hyde, K.D. and Bhat, D.J. 2014. Phylogenetic placement of Bahusandhika, Cancellidium and Pseudoepicoccum (asexual Ascomycota). Phytotaxa 176:68-80

Rappaz, F. 1987. Taxonomie et nomenclature des Diatrypacées à asques octosporés. Mycologia Helvetica, 2(3): 285-648.

Réblová, M. 2011. New insights into the systematics and phylogeny of the genus Jattaea and similar fungi of the Calosphaeriales. Fungal Diversity, 49:167-198.

Richardson, M.J. 2004. Coprophilous fungi from Morocco. Botanical Journal of Scotland, 56(2): 147-162. DOI: 10.1080/03746600408685075.

Rogerson, C.T. and Samuels, G.J. 1994. Agaricicolous Species of Hypomyces." Mycologia, 86(6): 839-66. DOI:10.2307/3760597.

Roumeguère, C. 1882. Fungi Gallici exsiccati. Centurie XXII. Revue Mycologique (Toulouse), 4(15): 150-159.

Roumeguère, C. 1885. Fungi exsiccati praecipue Gallici. Centurie XXXIV. Revue Mycologique, (Toulouse) 7 (26): 167-178.

Roumeguère, C. 1886. Fungi Gallici exsiccati - Centurie XXXVII. Revue Mycologique (Toulouse), 8 (30): 85-94.

Roumeguère, C. 1887. Fungi Selecti exsiccati praecipue Galliae et Algeriae - Centurie XLI. Revue Mycologique (Toulouse), 9(33,34) :18-29; 100-109.

Saccardo P.A. 1878. Enumeratio Pyrenomycetum Hypocreaceorum bucusque cognitorum. Michelia, 1: 277-355. 
Saccardo, P.A. 1882. Sylloge fungorum omnium hucusque cognitorum - Volume I. Berolini : Fratres Borntraeger, Padua (Italy), 768 pages.

Saccardo, P.A. 1883. Sylloge fungorum omnium hucusque cognitorum - Volume 2. Berolini : Fratres Borntraeger, Padua (Italy), 815 pages.

Saccardo, P.A. 1884. Sylloge fungorum omnium hucusque cognitorum - Volume 3. Berolini : Fratres Borntraeger, Padua (Italy), 860 pages..

Saccardo, P.A. 1886. Sylloge fungorum omnium hucusque cognitorum - Volume 4. Berolini : Fratres Borntraeger, Padua (Italy), 807 pages.

Saccardo P.A. 1891. Sylloge fungorum omnium hucusque cognitorum - Volume 9. Berolini : Fratres Borntraeger, Padua (Italy), 1141 pages.

Saccardo, P.A. 1892. Sylloge fungorum omnium hucusque cognitorum - Volume 10. Berolini : Fratres Borntraeger, Padua (Italy), 964 pages.

Saccardo, P.A. 1895. Sylloge fungorum omnium hucusque cognitorum - Volume 11. Berolini : Fratres Borntraeger, Padua (Italy), 753 pages.

Saccardo, P.A. 1899. Sylloge fungorum omnium hucusque cognitorum- Volume 14. 1316 pages

Saccardo, P.A. 1902. Sylloge fungorum omnium hucusque cognitorum - Volume 16. Berolini : Fratres Borntraeger, Padua (Italy), 1291 pages.

Saccardo, P.A. 1913. Sylloge fungorum omnium hucusque cognitorum - Volume 22. Berolini : Fratres Borntraeger, Padua (Italy), 1612 pages.

Saccardo, P.A. 1926. Sylloge fungorum omnium hucusque cognitorum - Volume 24 (Part1). Berolini : Fratres Borntraeger, Padua (Italy), 703 pages.

Saccardo, P.A. 1928. Sylloge fungorum omnium hucusque cognitorum - Volume 24 (Part 2). 732 pages.

Saccardo, P.A. 1931. Sylloge fungorum omnium hucusque cognitorum - Volume 25. 1093 pages.

Saccardo, P. A. and Berlese, A. N. 1886. Fungi Algerienses. - a Claro prof. L. Trabut lecti. Revue mycologique, 29: 33-37.

Samuels, G.J. and Blackwell, M. 2001. Pyrenomycetes-fungi with perithecia. In: McLaughlin DL, McLaughlin EG, Lemke PA (eds) The Mycota- systematics and evolution. VII part A. Springer, Verlag,pp 221-255.

Semenova, E.F., Shpichka, A.I. and Moiseeva, I.Y. 2012. About essential oils biotechnology on the base of microbial synthesis. Pharm Sci 4:29-31.

Smahi, H., Belhoucine-Guezouli, L., Bouhraoua, R.T., Franceschini, A. and Linaldeddu, B.T. 2018. First Report of Branch Canker and Dieback Caused by Cryphonectria naterciae on Quercus suber in Algeria. Plant disease 102(1): 251.

Stadler, M., Læssøe, T., Fournier, J., Decock, C., Schmieschek, B., Tichy, H-V., Peršoh, D. (2014) A polyphasic taxonomy of Daldinia (Xylariaceae). Studies in Mycology, 77: 1143. DOI: $10.3114 / \operatorname{sim} 0016$.

Steinheil, A. 1834. Matériaux pour servir à la flore de Barbarie. II : Notice sur les cryptogames recueillies aux environs de Bone. Annales des Sciences Naturelles (série Botanique), 2(1): 282-289. 
Stevenson, J.A. 1926. Foreign Plant Diseases. A Manual of Economic Plant Diseases which are New to or Not Widely Distributed in the United States. United States Department of Agriculture, Washington DC, USA. 196 pages.

Sung, G-H., Hywel-Jones, N.L., Sung, J-M., Luangsaard, J.J., Shresthra, B. and Spatafora, J.W. 2007. Phylogenetic classification of Cordyceps and the clavicipitaceous fungi. Stud Mycol 57:5-59.

Sydow, H. and Sydow, P. 1915. Novae fungorum species. Annales Mycologici. 13(1):35-43.

Sydow, P. and Sydow, H. 1924. Monographia Uredinearum. 4:1-671.

Tlemsani, M. 2010. Contribution à l'étude du flétrissement vasculaire du pois chiche (Cicer arietinum L.) causé par Fusarium oxysporum Schelcht. Emend. Snyd. \& Hans. f. sp. ciceri (Padwick): caractérisation, lutte biologique et comportement variétal. Master thesis, Université Ahmed Ben Bella, Oran (Algeria), 95 pages.

Tligui, H., Aoufi, S. and Agoumi, A. 2006. Mycétome du creux poplité à Madurella mycetomatis: à propos d'un cas. Journal de Mycologie Médicale (Paris),16(3): 173-176.

Trabut, L. 1898. Destruction de l'altise de la vigne par un champignon parasite (Sporotrichum globuliferum ou Isaria globulifera). Revue de viticulture, 5:13-27.

Trabut, L. 1907. Un Champignon parasite du Parlatoria zizyphi (Microcera Parlatorice sp. nov.). Bulletin, agricole de l'Algérie et de la Tunisie (Alger), 19 :11-23.

Vanev, S.G. 1996. Fungi of the genus Discosia (Deuteromycotina) in the Mediterranean area. Bocconea, 5: 351-357.

Vu, D., Groenewald, M., de Vries, M., Gehrmann, T., Stielow, B., Eberhardt, U., Al-Hatmi, A., Groenewald, J.Z., Cardinali, G., Houbraken, J., Boekhout, T., Crous, P.W., Robert, V. and Verkley, G.J.M. 2019. Large-scale generation and analysis of filamentous fungal DNA barcodes boosts coverage for kingdom fungi and reveals thresholds for fungal species and higher taxon delimitation. Studies in Mycology, 92: 135-154. DOI: 10.1016/j.simyco.2018.05.001.

Wijayawardene, N.N., Hyde, K.D., Rajeshkumar, K.C. et al. 2017. Notes for genera: Ascomycota. Fungal Diversity, 86(1): 1-894. DOI: 10.1007/s13225-017-0386-0.

Woudenberg, J.H.C., Meijer, M., Houbraken, J. and Samson, R.A. 2017. Scopulariopsis and Scopulariopsis-like species from indoor environments. Stud. Mycol. 88: 1-35.

Wraight, S.P., Carruthers, R.I., Bradley, C.A., Jaronski, S.T., Lacey, L.A., Wood, P. and GalainiWraight, S. 1998. Pathogenicity of the entomopathogenic fungi Paecilomyces spp. and Beauveria bassiana against the silver leaf white fly, Bemisia argentifolii. J Invertebr Pathol 71:217-226.

Xu, J., Yang, X. and Lin, Q. 2014. Chemistry and biology of Pestalotiopsis derived natural products. Fungal Divers 66:37-68.

Yezli, W., Hamini-Kadar, N., Zebboudj, N., Blondin, L., Tharreau, D. and Kihal, M. 2019. First report of crown and root rot of tomato caused by Fusarium equiseti in Algeria Journal of Plant Pathology, 101(4): 1249. DOI: 10.1007/s42161-019-00311-9.

Youcef-Khodja, L. 2010. Contribution à l'inventaire et à la connaissance des macrochampignons dans le Parc National de Chréa; impacts des facteurs écologiques Master Thesis, Université des Sciences et de la Technologie Houari Boumediène, Alger (Algeria), 120 pages. 
Zait, H., Madani, K., Abed-Benamara, M., Achir, I. and Hamrioui, B. 2008. Mycetoma in Algeria. About two new cases. Review of cases reported between 1995 and 2005. J Mycol Med;18: 116-22.

Zamoum, M., Berchiche, S., Sai, K., Triggia, O. and Tarasco, E. 2011. Preliminary Survey of the Occurrence of Entomopathogenic Nematodes and Fungi in the Forest Soils of Algeria. Silva Lusitana, 19.

Zhang, N., Castlebury, L.A., Miller, A.N., Huhndorf, S.M., Schoch, C.L., Seifert, K.A., Rossman, A.Y., Rogers, J.D., Kohlmeyer, J., Volkmann-Kohlmeyer, B. and Sung, G.H. 2006. An overview of the systematics of the Sordariomycetes based on four-gene phylogeny. Mycologia 98:1076-1087. 